\title{
Preventive Antibiotic Treatment Increases Neonatal Chicks Susceptibility to Salmonella Infection Via Disrupting Gut Microbiota and Linoleic Acid Metabolism
}

\section{Xueran Mei}

Sichuan University https://orcid.org/0000-0001-8382-562X

Boheng Ma

Sichuan University

Xiwen Zhai

Sichuan University

Anyun Zhang

Sichuan University

Changwei Lei

Sichuan University

Lei Zuo

Sichuan University

Yizhi Tang

Sichuan University

Xin Yang

Sichuan University

\section{Yufeng Gao}

Sichuan University

Changyu Zhou

Sichuan University

Hongning Wang ( $\nabla$ whongning@163.com )

Sichuan University https://orcid.org/0000-0002-7244-9929

\section{Research}

Keywords: Neonatal chicks, Gut microbiota, Florfenicol, Gut colonization, Salmonella enterica serovar Enteritidis, Conjugated linoleic acid, 12,13-diHOME

Posted Date: September 22nd, 2020 
DOI: https://doi.org/10.21203/rs.3.rs-77489/v1

License: (c) (i) This work is licensed under a Creative Commons Attribution 4.0 International License. Read Full License 


\section{Abstract}

Background: Antimicrobial agents have been widely used in animal farms to prevent and treat animal diseases. However, antimicrobial agents may change the bacterial community and increase susceptibility to the pathogenic bacteria infection. Here, we used metagenomic and metabonomic approach to investigate the effects of florfenicol (FFC) pre-treatment on colonization of Salmonella enterica serovar Enteritidis (S. Enteritidis) in intestines of neonatal chicks through analysis of host responses, microbiota and metabolic changes.

Results: We observed that FFC pre-treatment significantly increases the level of $S$. Enteritidis in the cecal contents, spleen and liver and also induces changes to the cecal microbiota and metabolism. Prior to $S$. Enteritidis infection, FFC significantly reduced the content of Lactobacillus, and significantly affected the linoleic acid metabolism pathway, including significantly reducing the levels of conjugated linoleic acid (CLA), and significantly increasing the abundance of 12,13-EpOME and 12,13-diHOME in cecum. After infection with $S$. Enteritidis, the abundance of Proteobacteria were significantly increased and the Salmonella-induced intestinal inflammatory responses and intestinal barrier damage were exacerbated. Supplementation with CLA could maintain intestinal integrity, reduce intestinal inflammation, and directly inhibit Salmonella growth to effectively reduce the Salmonella colonization, whereas the 12,13-diHOME through promoting intestinal inflammation and destroying the intestinal barrier function to support the Salmonella infection.

Conclusions: Overall, FFC can decrease levels of Lactobacillus and CLA, and elevate cecal 12,13-diHOME concentrations in neonatal chicks and thereby increases susceptibility to Salmonella infection. This study revealed a potential health impact of antibiotics and disturbed gut microbiota and linoleic acid metabolism might be an intestinal health-impairing attribute and may contribute to Salmonella colonization.

\section{Background}

The global human population will reach 8.5 billion by 2030, which rising high demand for international livestock products [1]. Chickens are the most abundant livestock in the world, which more than 60 billion chickens are produced annually, with production predicted to increase further in the next 20 years $[2,3]$. The health of chickens is essential to ensure the safety of poultry products and effective control and management of disease. Salmonella enterica is a major medical and economic problem worldwide, and causes significant morbidity and mortality in poultry $[4,5]$. The Salmonella colonization in chickens is of particular importance because the association of this pathogen with poultry products is considered to be a significant source of infection to humans, is the leading cause of foodborne disease outbreaks worldwide [6-9].

Microbiota-mediated mechanisms of colonization resistance is critical to prevent pathogens invasion in some niches within animal hosts [10]. Particularly, the intestine is best site to characterized the 
mechanisms of colonization resistance because the intestinal lumen contains trillions of commensal microbes and those microbes are interact closely with their hosts as well as each other in intricate microbial networks $[11,12]$. The microbial community of the chicken intestine is highly diverse with more than 1000 species of bacteria and the population density can be up to about $10^{11} \mathrm{cells} / \mathrm{g}$ intestinal contents, which can protect chickens from the infection $[13,14]$. The gut microbiota helps protect chickens from colonization by zoonotic pathogens, but this can be weakened through antibiotics administration that disturb the gut bacterial community and metabolism [15]. The large-scale intensive rearing systems usually containing flocks of 20,000 birds or more, which depend on antibiotics to prevent and treat animal disease [16]. In China, the veterinary antibiotics accounted for $84.3 \%$ (pig: $52.2 \%$, chicken: $19.6 \%$, and other animals: $12.5 \%$, whereas the human antibiotics only shared $15.6 \%$ [17]. And it is estimated that the global consumption of antibiotics used for chickens, pigs, and cattle will increase by $67 \%$, from 63,151 tons in 2010 to 105,596 tons in 2030 [18]. Consequently, disruptions of the microbiota composition induced by antibiotics lead to disorder in the ecological balance between microbes and host, dramatically reduces the colonization resistance and enhances the susceptibility to infection by various pathogens [19-21]. In oral administration infection models in mice, antibiotic pretreatment allows efficient colonization of the cecum and colon by $S$. enterica serovar Typhimurium, and enhances Salmonella expansion and fecal shedding $[22,23]$.

Furthermore, the neonatal chicks exhibit highly susceptibility to infections with Salmonella serovars because of their immature gut microbiota, and the complexity of the neonate gut microbiota gradually increases from day 1 to day 19 of life [24,25]. Although the chickens are coprophagic and transfer of cecal microbiota from adult chickens to neonatal chicks increases resistance to Salmonella infection [26, 27]. Unlike other farm animals, the neonatal chicks are hatched in the clean environment of a hatchery without any contact with adult chickens and their colonization resistance is only dependent on the environment [28]. If a pathogen appears in the environment, the immature gut microbiota of the newly hatched chick enabling such a pathogen essentially unrestricted multiplication. Therefore, the large-scale intensive rearing systems usually depend on antibiotics to prevent and control the disease outbreaks, which makes the gut microbiota of chicks disrupted and may lead to the chicks are more susceptible to Salmonella infection.

We hypothesize that the newly hatched chicks are pre-treated with an antibiotic accompany changes with the disorders of the gut microbiota and metabolic profile, which lead to the chicks are more susceptible to Salmonella infection. Therefore, it is necessary to better understand how antibiotics affect the mechanism of colonization resistance. The objectives of the study were to investigate the influence of a 7-day treatment course of florfenicol, a most commonly used broad-spectrum antibiotic in poultry in many countries $[17,18,29]$, on the composition of the microbiota community and metabolic profile of the neonatal chicks, and find the potential factors enable support $S$. Enteritidis growth in the cecum of neonatal chicks.

\section{Methods}




\section{Bacterial strains}

The Salmonella enterica serovar Enteritidis (S. Enteritidis, ATCC 13076) floR mutant strain was used as the challenge strain. The $S$. Enteritidis floR mutant was constructed using the plasmid homologous recombination integration method as previously described [30]. Briefly, the sequence of floR gene (NG_047860.1) was synthesized and cloned into E. coli Cloning vector. Then the upstream and downstream homologous recombinant arms were amplified by PCR from $S$. Enteritidis genome use ultrafidelity DNA polymerase, and floR sequence was amplified by PCR from template vector. The fragments were assembled by the Fusion PCR to construct the gene targeting fragment, and the PCVD442 suicide plasmid was digested with restriction endonuclease to construct gene target plasmid pCVD442-floR,

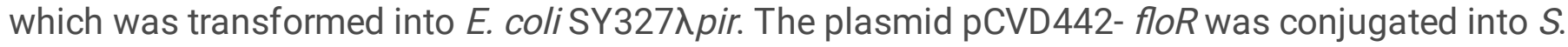
Enteritidis using E. coliSY327 $\lambda$ pir as a donor strain and plated on florfenicol (FFC) resistant chromogenic xylose lysine tergitol 4 (XLT4) agar plate to select for positive clones that had integrated the suicide plasmid. Finally, a colony that was FFC resistant verified by PCR and gene sequencing. Prior to inoculation, S. Enteritidis was grown overnight in Luria-Bertani broth at $37^{\circ} \mathrm{C}$ with shaking at $200 \mathrm{rpm}$.

\section{Chicken, florfenicol intervention and infection}

Leghorn layer chicks (1-day-old) were hatched from the same batch eggs of specific-pathogen-free (SPF) birds (Beijing Boehringer Ingelheim Vital Biotechnology Co., Ltd., China), and each assigned group was reared in an individual GJ-1 SPF isolator (Suzhou Fengshi Laboratory Animal Equipment Co., Ltd., China). Animals were received non-medicated chick feed and water ad libitum and raised under controlled environmental conditions with a 16-h lighting cycle and a temperature of $32^{\circ} \mathrm{C}$ at day 1 which was gradually reduced and maintained at $24^{\circ} \mathrm{C}$ on day 10 .

\section{Animal protocol 1: Effect of florfenicol pre-treatment on intestinal Salmonella colonization. Eighty-eight} newly hatched chicks were assigned at random to four groups, each group included 22 chicks in three time points ( $\mathrm{n}=7$ to 8 chicks in each time point) and treated with a 7-day antibiotic treatment $(30 \mathrm{mg} / \mathrm{kg}$ b. w.) of florfenicol (FFC) or infected with $\sim 10^{8} \mathrm{cfu}$ of the challenge strain $S$. Enteritidis by oral gavage. The treatments were as follows: (1) NT, control group neither FFC-treated nor $S$. Enteritidis-infected; (2) FT, FFC-treated group; (3) ST, S. Enteritidis-infected group; (4) and FST, FFC-pre-treated and S. Enteritidisinfected group. On days 11,18 and 25 , the chicks were euthanized for analysis.

\section{Animal protocol 2. Effect of conjugated linoleic acid (CLA) and 12,13-diHOME on intestinal Salmonella colonization. Forty newly hatched chicks were assigned at random to four groups ( $n=10$ chicks per} group) were treated with the CLA or 12,13-diHOME by gastric gavage. The CLA (purity: $>99 \%$, Nu-Chek Prep, Elysian, MN, USA) is the mixture of $65.5 \% \mathrm{c} 9, \mathrm{t} 11$-CLA and $34.5 \% \mathrm{t} 10, \mathrm{c} 12-\mathrm{CLA}$ isomers by LC-MS detection (Data not showed), and the 12,13-diHOME (purity: $\geq 98 \%$, Cayman Chemical, Ann Arbor, Michigan, USA) solution was prepared according to the previous study [31]. The dose of CLA and 12,13diHOME is corresponds to the amount of eaten diet supplemented with 1\% CLA and 12,13-diHOME (1\% [10 mg/g diet] $\times 3-9$ [gram of diet eaten in average by chicks for 1-7 day]). And the chicks were divided 
into four groups which included: (1) NT, control group; (2) ST, S. Enteritidis-infected group; (3) CLA, CLApre-treated and $S$. Enteritidis-infected group; and 4) 12,13-diHOME, 12,13-diHOME-pre-treated and $S$. Enteritidis-infected group. On days 11, the chicks were euthanized for analysis.

After chicks were euthanized, the cecal contents and internal organs were aseptically collected and homogenized using the PBS. For enumerating Salmonella loads, an aliquot (100 $\mu$ l) of appropriate dilutions was spread onto XLT4 agar plates ( $50 \mathrm{ug} / \mathrm{ml}$ florfenicol), which Salmonella appearing typical black colonies after incubation at $37^{\circ} \mathrm{C}$ for $24 \mathrm{~h}$.

\section{Histopathology and microscopic analysis of the intestine}

Parts of ileal tissue were perfusion-fixed with formalin for $24 \mathrm{~h}$. After gradient dehydration with ethanol, specimens were embedded in paraffin. Subsequently, $5 \mu \mathrm{m}$ sections were rehydrated and stained with Alcian blue. Representative images were obtained by a BA400 digital microscope (Motic Group CO., LTD., China). The mean density was calculated by the integral optical density and area of the positive Alcian blue staining using the Image-Pro ${ }^{\circledR}$ Plus v6.0 analysis system (Media Cybernetics, USA), and significance of differences was determined by a one-way ANOVA. A $P$-value of less than 0.05 was considered statistically significant. To determine the degree of lesion, pathological score was monitored as previously described [32]. And the scanning electron microscope (SEM) (Inspect ${ }^{\mathrm{TM}}$, FEI Ltd., USA) of the intestinal villi was conducted as previously described [33].

\section{DNA extraction, 16S rRNA gene sequencing and data analysis}

Seven or eight chicks per treatment were randomly chosen at three different time points, 11,18 , and 25 days of age, and euthanized by carotid artery bleeding. The cecal contents were collected within 5 min of euthanasia, immediately placed in pre-cooling cryogenic vials, and stored at $-80^{\circ} \mathrm{C}$ until DNA extraction. Total genomic DNA was extracted from cecal contents using the QIAamp DNA Stool Mini Kit (Qiagen, Germany) according to manufacturer's protocols, and stored at $-20^{\circ} \mathrm{C}$ prior to further analysis. The concentration and quality of extracted DNA samples were measured by Nanodrop 2000 (Thermo Fisher Scientific, Waltham, MA, United States) and agarose gel electrophoresis, respectively.

Using the isolated genomic DNA as the template, the V3-V4 hypervariable regions of the bacterial $16 \mathrm{~S}$ rRNA genes were PCR-amplified with primers 338F (5'-ACTCCTACGGGAGGCAGCA-3') and 806R (5'GGACTACHVGGGTWTCTAAT-3') following the method previously described [34]. Amplicons were then sequenced on the Illumina MiSeq platform (Illumina Inc., USA) using $2 \times 250$ bp cycles. These sequence data are deposited in the NCBI database within the Bioproject PRJNA655362 under the SRA study SRP277009. The QIIME was employed to process the sequencing data. Briefly, raw sequencing reads with exact matches to the barcodes were assigned to respective samples and identified as valid sequences. The low-quality sequences were filtered through following criteria [35, 36]: sequences that had a length of $<150 \mathrm{bp}$, sequences that had average Phred scores of $<20$, sequences that contained ambiguous bases, and sequences that contained mononucleotide repeats of $>8 \mathrm{bp}$. Paired-end reads were assembled using FLASH [37]. After chimera detection, the remaining high-quality sequences were 
clustered into operational taxonomic units (OTUs) at $97 \%$ sequence identity by UCLUST [38]. A representative sequence was selected from each OTU using default parameters. OTU taxonomic classification was conducted by BLAST searching the representative sequences set against the Greengenes Database [39] using the best hit [40]. An OTU table was further generated to record the abundance of each OTU in each sample and the taxonomy of these OTUs. OTUs containing less than $0.001 \%$ of total sequences across all samples were discarded. To minimize the difference of sequencing depth across samples, an averaged, rounded rarefied OTU table was generated by averaging 100 evenly resampled OTU subsets under the $90 \%$ of the minimum sequencing depth for further analysis.

Sequence data analyses were mainly performed using QIIME and R packages (v3.2.0) [41]. OTU-level alpha diversity indices, such as Shannon indices, species abundance and Pielou indices were calculated using the OTU table in QIIME. Beta diversity analysis was performed to investigate the structural variation of microbial communities across samples using Bray-Curtis distances metrics and visualized via principal coordinate analysis (PCOA). The taxonomy compositions and abundances were visualized using MEGAN [42] and GraPhIAn [43]. Linear discriminant analysis effect size (LEfSe) was performed to detect differentially abundant taxa across groups using the default parameters [44].

\section{Quantitative PCR for microbiota analysis}

Bacterial composition of the microbiota was measured by qPCR as previously described [45-48]. All qPCR reactions were performed using the Bio-Rad real-time PCR detection system (Bio-Rad CFX Maestro 1.1, 3.0, USA) and SsoFast EvaGreen Supermix (Bio-Rad Inc., USA) and tested according to the manufacturers' instructions. The extracted genomic DNA in the cecal content was used as a template for qPCR using the main group-specific primers (Supplementary Table 1): All eubacteria, Lactobacillus, Bacteroidetes, Enterobacteriaceae, Clostridium butyricum and Faecalibacterium prausnitzii. Serial dilutions of plasmids containing the target gene cloned into the pMD-19 T cloning vector (TaKaRa, Dalian, China) were analyzed to generate a standard curve and calculate absolute counts of the target gene.

\section{Metabolomics for chicken cecal content}

Untargeted metabolomics. Chickens were sacrificed, and the cecum was resected. The cecal contents were obtained and stored at $-80^{\circ} \mathrm{C}$ until use for the metabolomics analysis. The method of sample preparation for LC/MS detection are previously described [49]. Briefly, $50 \mathrm{mg}$ freeze-dried sample, $800 \mu \mathrm{l}$ methanol, and $5 \mu \mathrm{LL}$-o-Chlorophenylalanine (internal standard) were added to a $1.5 \mathrm{~mL}$ Eppendorf tube. And all the samples were grinded to fine powder using grinding mill at $65 \mathrm{HZ}$ for $90 \mathrm{~s}$ followed by being vortexed for $30 \mathrm{~s}$, and centrifuged at $12,000 \mathrm{rpm}, 4^{\circ} \mathrm{C}$ for $15 \mathrm{~min}$. Then $200 \mu \mathrm{L}$ of supernatant was transferred to a new vial for LC-MS analysis. A total of $10 \mu \mathrm{l}$ of the sample solution at $4{ }^{\circ} \mathrm{C}$ were injected to the LC-MS system (Thermo, Ultimate 3000LC, Exactive Orbitrap) with an Agilent C18 column (Hypergod C18, $100 \times 2.1 \mathrm{~mm} 1.9 \mu \mathrm{m}$ ) and the column temperature was maintained at $40^{\circ} \mathrm{C}$. The mobile phase consisted of solution A and B [A was $0.1 \%$ formic acid $/ 5 \%$ acetonitrile/water $(\mathrm{v} / \mathrm{v} / \mathrm{v})$ and $B$ was $0.1 \%$ formic acid/acetonitrile ( $\mathrm{v} / \mathrm{v})]$ and the flow rate was $350 \mu \mathrm{l} / \mathrm{min}$. The gradient was set as follows: 
$0 \% \mathrm{~B}$ at $0 \mathrm{~min}, 20 \% \mathrm{~B}$ at $1.5 \mathrm{~min}, 100 \% \mathrm{~B}$ at $9.5 \mathrm{~min}$ to $14.5 \mathrm{~min}$, and $0 \% \mathrm{~B}$ at $14.6 \mathrm{~min}$ to $18 \mathrm{~min}$. Samples were analyzed in positive and negative ion modes using $300^{\circ} \mathrm{C}$ of heater temperature, $350{ }^{\circ} \mathrm{C}$ of capillary temperature, and 3.0 KV of spray voltage. The flow rates of sheath gas, aux gas, and sweep gas were 45 , 15 , and 1 arb, respectively. The peaks were aligned according to the $\mathrm{m} / \mathrm{z}$ value and normalized migration time. The peak areas were calculated by normalizing against the internal standards, and the metabolites were identified searching against the database based on the $\mathrm{m} / \mathrm{z}$ value and normalized migration time. Compound Discoverer Software (Thermo) was used to process the Thermo RAW files, and the data after editing were performed Multivariate Analysis using SIMCA-P 14.0 software (Umetrics AB, Umea, Sweden). Metabolites selected as biomarker candidates for further statistical analysis were identified on the basis of variable importance in the projection (VIP) threshold of 1 from the sevenfold cross-validated OPLS-DA model, which was validated at a univariate level with adjusted $\mathrm{P}<0.05$. And the MetaboAnalyst (version 3.0) was used for the identification of metabolic pathways [50].

Targeted metabolomics. $50 \mathrm{mg}$ dried cecal contents and $800 \mu \mathrm{l}$ methanol were added to a $1.5 \mathrm{~mL}$ Eppendorf tube. And the sample was grinded to fine powder using grinding mill at $65 \mathrm{HZ}$ for $90 \mathrm{~s}$ followed by being vortexed for $30 \mathrm{sec}$, and centrifuged at 12,000 rpm, $4^{\circ} \mathrm{C}$ for $15 \mathrm{~min}$. Next, the $200 \mu \mathrm{l}$ of supernatant was taken for detection.

For quantitative detection of linoleic acid and CLA, $1 \mu$ of each sample was injected onto a DB-5 column (60 m x $0.25 \mathrm{~mm} 0.25 \mu \mathrm{m})$ using Thermo Trace $1300 \mathrm{GC}$ (Thermo Fisher Scientific, USA) system online with mass spectrometer (ISQ7000, Thermo Fisher Scientific, USA) (GC-MS). The temperature program was as follows: the initial oven temperature $140^{\circ} \mathrm{C}$ was hold for $5 \mathrm{~min}$, then programmed to increase with $10^{\circ} \mathrm{C} / \mathrm{min}$ to $180^{\circ} \mathrm{C}$, with $4^{\circ} \mathrm{C} / \mathrm{min}$ to $210^{\circ} \mathrm{C}$, to reach finally with $10^{\circ} \mathrm{C} / \mathrm{min} 260^{\circ} \mathrm{C}$ and hold for $20 \mathrm{~min}$. Helium (99.999\% purity) was used as carrier gas with a flow rate of $1.5 \mathrm{ml} / \mathrm{min}$. The MS inlet line and the ion source temperatures were maintained at 260 and $230^{\circ} \mathrm{C}$, respectively, and the MS ionization energy was $70 \mathrm{eV}$. A full scan mode set from $5 \mathrm{~min}$ to $20 \mathrm{~min}$, monitoring $\mathrm{m} / \mathrm{z}$ range from 33 to 550 Da, was used for the identification of possible interferences from the matrix extract.

For quantitative detection of 12,13-EpOME and 12,13-diHOME, $4 \mu \mathrm{l}$ of each sample was injected onto an Acquity UPLC BEH C18 column $(100 \mathrm{~mm}$ x $2.1 \mathrm{~mm}$ x $1.7 \mu \mathrm{m})$ using an Acquity UPLC (Waters Corporation, USA) coupled with triple quadrupole mass spectrometry (API5500, AB SCIEX LLC., USA) (UPLC-QqQ-MS). The mobile phase consisted of solution $A$ and $B$ ( $A$ was water and $B$ was acetonitrile) and the flow rate was $300 \mu \mathrm{l} / \mathrm{min}$. The gradient was set as follows: 10\% B at $0 \mathrm{~min}, 10 \% \mathrm{~B}$ at $1.0 \mathrm{~min}, 90 \% \mathrm{~B}$ at $1.5 \mathrm{~min}$, $90 \%$ B at $5.0 \mathrm{~min}, 10 \%$ B at $6.0 \mathrm{~min}, 10 \%$ B at $7.0 \mathrm{~min}$. Samples were analyzed in negative ion modes using $550{ }^{\circ} \mathrm{C}$ of atomizing temperature, $-4.5 \mathrm{KV}$ of spray voltage, and MRM reaction monitoring of scanning method. The flow rates of curtain gas, collision gas, GS1 (atomizing gas) and GS2 (auxiliary gas) were $35,9,55$, and 55 arb, respectively.

\section{RNA isolation and RT-qPCR of the cytokines from tissue for expression analysis.}

Total RNA from the ileum and liver tissue was extracted by using Trizol reagent (Invitrogen Life Technologies, Carlsbad, CA) according to the manufacturer's instructions. The quality and concentration 
of RNA were measured using the Nanodrop 2000 spectrophotometer. $1 \mu \mathrm{g}$ of total RNA from each sample was reverse transcribed into cDNA using the SuperScript II (Invitrogen Life Technologies, Carlsbad, USA). The primers used for the reverse transcription were oligo (dT) primer and random hexamers. The quantitative PCR reaction was performed with the SsoFast EvaGreen Supermix using a Bio-Rad CFX realtime PCR detection system following the manufacturer's protocols. Primers used in this study were listed in Supplementary Table $2[51,52]$. Relative mRNA expression levels of each target gene (ZO-1, Occludin, Claudin 3, MUC2, TFF2, IL-22, IL-17A, INF-a, CYP1A2, EPHX2 and GAPDH) were calculated using the log2 of the fold change method. The sample was run in triplicate parallel reactions in all samples.

\section{ELISA detection}

The concentrations of chicken IL-1 $\beta$, IL-6, IL-8, IL-10, INF- $\gamma$, TNF- $\alpha$ in the ileum tissue, serum IgG, LPS, diamine oxidase (DAO), D-lactate and intestinal mucosal secretory immune globulin A (SIgA) were determined using the mlbio enzyme-linked immunosorbent assay (ELISA) kit (Shanghai Enzyme-linked Biotechnology Co., Ltd., China) according to the manufacturer's instructions. Their concentrations were then calculated from the standard curves.

\section{Data and statistical analysis}

The heatmap of the interrelationship between the differential flora and the metabolites was drawn using $R$ (3.6.1) pheatmap package. The correlation coefficient calculated by heat map $(R<0.5)$ was used to exclude the metabolites and flora with weak correlation and no correlation, and Cytoscape (3.7.1) software was used to draw the correlation network diagram, which the flora and metabolites were used to form points, and the line segment represented the correlation size. The distribution of bacterial communities and their potential correlations with differential metabolites were determined using canonical correspondence analysis (CCA), using the R (3.6.1) vegan package. Statistical analyses were conducted with SPSS 20.0 (SPSS Inc., USA). The data collected are presented geometric median or mean \pm standard deviation. Statistical significance was determined by Mann-Whitney test or One-way ANOVA. Mann-Whitney test was used for comparing two groups. One-way ANOVA with a Dunnett's multiple comparison test was used for pair-wise comparison of means from more than two groups in relation to the control group. The $p$ values of less than 0.05 were considered statistically significant $\left({ }^{*} p<0.05 ;{ }^{*} p<\right.$ $0.01 ; * \star \star p<0.001)$.

\section{Results}

\section{Florfenicol exposure increases susceptibility to $S$. enteritidis infection}

We established a study design (Fig. 1a) in which SPF chicks were fed either the FFC-treated (FT), the $S$. Enteritidis-infected (ST) or simultaneous treatment of $S$. Enteritidis and FFC (FST). Under the SPF environment, all chicks were cultured negative for Salmonella spp. until experimental infection with $S$. enteritidis and control group remained culture negative for Salmonella spp. throughout the study. The colonization and translocation of $S$. enteritidis in the intestinal tract of chicks directly determines the 
survival and pathogenicity of $S$. enteritidis. Therefore, we examined $S$. enteritidis levels in the caecum, spleen and liver and found that the antibiotic (FFC) could promote $S$. enteritidis colonization and translocation in the intestines of chicks. The number of $S$. enteritidis (log10 CFU/g tissue) significantly increased by $25.49 \%$ (Cecal contents, $P<0.01$ ), 23.04\% (Spleen, $P<0.01$ ) and $21.33 \%$ (Liver, $P<0.01$ ), respectively, in the FST group compared with those in the ST group at 3 days post-infection (dpi). Similar results were observed at day 18 (10 dpi) and day 25 (17 dpi), although their Salmonella loads are less than the $3 \mathrm{dpi}$ (Fig. 1b). The above results indicate a robust influence of antibiotic upon susceptibility to oral $S$. enteritidis infection in neonatal chicks.

\section{Florfenicol administration aggravates $S$. enteritidis-induced morphology and intestinal barrier function injury}

The FFC intervention made the chicks more susceptible to Salmonella infection. It is possible that antibiotics disrupted the immature intestinal barrier homeostasis of the chicks, thus changing the intestinal permeability and making the chicks carrying more Salmonella in their internal organs. Therefore, we investigated the effects of FFC administration on $S$. enteritidis-induced intestinal morphology injury. H\&E staining showed that the NT group exhibited an intact structure of the ileal mucosa, neat intestinal villi, deep crypts, and a clear and complete gland structure, as also observed in the FT group (Additional file 1: Figure S1a, b). The ST group showed that the structure of the ileal mucosa was incomplete, villi had a shorter length and sparse distribution, and the crypts were shallow (Additional file 1: Figure S1c). However, the FST group increased loss of mucosal structures, and atrophic crypts as well as lamina propria bowel edema can be observed (Additional file 1: Figure S1d). The histological injury score (Additional file 1: Figure S1e) was assessed based on the H\&E staining images, and the score showed quantifiable results of tissue damage. The score of the chicks in the ST group $(7.13 \pm 0.44)$ was significantly higher than normal chicks $(0.63 \pm 0.18)$. Compared with the ST group, the FFC preadministration $(10.75 \pm 0.45)$ significantly increased the injury score of the ileum. We also utilized SEM to examine the intestinal structure in different groups. The results showed that the NT group had complete ileal villi, which formed full and closely arranged structures (Fig. 2e), the FT group also had intact ileal villi, but the arrangement structure was relatively loose (Fig. 2f). As expected, the ileal villi in the ST group were damaged (Fig. 2g), whereas those in FST group showed more severely (Fig. 2h). These results suggest that, although FFC has less effect on intestinal morphology, it can aggravate the intestinal morphological damage in the presence of Salmonella invasion.

The effect of FFC on intestinal barrier function changes in ileum after $S$. enteritidis infection were also examined. We found that FFC can exacerbate the $S$. enteritidis-induced lleum permeability increase (Fig. 3a-d). The serum DAO and LPS levels in the FT group were significantly $(P<0.001$ and 0.05 respectively) higher than those in the NT group (Fig. 3c, d). In the case of Salmonella infection, serum D-lactate, DAO and LPS levels in both ST and FST group were significantly $(P<0.001)$ increased compared with that in NT group. However, FFC treatment significantly $(P<0.001)$ increased the serum D-lactate, DAO and LPS contents exposed to Salmonella infection (Fig. 3b-d). Alcian blue staining indicated that FFC significantly $(P<0.05)$ decreased the acidic mucin of ileum compared with NT group, as evident by the quantitative 
evaluation of positive Alcian blue staining (Fig. 2a, b) using the integral optical density measurement (Fig. 3a). Similarly, FFC treatment also significantly decreased $(P<0.01)$ mean density of acidic mucin (Fig. 2d; Fig. 3a) exposed to Salmonella infection. Transcriptional analysis of a range of relevant intestinal barrier genes was used to determine changes between these groups (Fig. 5). FFC treated significantly altered the gene transcription (Caludin1, IL-17A, IFN-a) in FT group. However, in the case of Salmonella infection, the FFC significantly reduced the expression of ZO-1, Occludin, Caludin1, MUC2 and TFF2, and significantly increased the expression of IL-17A, IL-22 and IFN-a. Furthermore, treatment with FFC significantly $(P<0.01)$ decreased SIgA secretion, but had no effect on serum IgG (Fig. 3e, $f)$. Nevertheless, FFC treated reduced the SIgA secretion more seriously $(P<0.001)$ after Salmonella infection (Fig. 3f). These results indicate that FFC intervention, to some extent, increased the intestinal mucosal permeability of chicks, reduced mucosal immunity and significantly increased the degree of damage to the intestinal mucosal barrier after Salmonella infection.

The levels of Salmonella colonization are tightly interconnected with the trigger of intestinal inflammation [53]. This suggested that the increased colonization levels might be linked to increased mucosal inflammation. Indeed, FFC treated chicks featured higher levels of gut inflammation after Salmonella infection (Fig. 4). This was verified by quantitative analysis of proinflammatory cytokines and antiinflammatory cytokines in ileum tissue. Salmonella infection after FFC-pretreated significantly increased the level of the cytokines IL-1 $\beta$ (Fig. 4a), IL-6 (Fig. 4b), IL-8 (Fig. 4c), TNF-a (Fig. 4e), and IFN-Y (Fig. 4f), whereas IL-10 (Fig. 4d) was significantly decreased. Moreover, the inflammatory cytokines (IL-1 $\beta$, IL-6, TNF- $a$ and IFN- $\gamma$ ) were also significantly increased in the FT group. These results indicate that the FFC exacerbated the Salmonella-induced inflammatory response. It is possible that antibiotic-treated causes Gram-negative bacterium releases LPS, which promotes intestinal inflammation, and this can be confirmed by serum LPS levels. Moreover, FFC may also shifts the gut microbiota and metabolic profiling of neonatal chicks, causing microbiotic and metabolic disorders and support Salmonella colonization.

\section{Florfenicol administration alters the gut microbiota}

The composition and density of the gut microbiota play an important role in combating Salmonella invasion, and oral pretreatment with antibiotics decreases colonization resistance and leads to an obviously post-antibiotic expansion of the Salmonella loading in the gut [53]. Thus, we hypothesized that the more Salmonella population observed in FFC-treated group might be linked to a disorder of the microbiota composition and density. To this end, comparative microbiota analysis of the cecal content of different groups at three different stages of infection (Day 3, 10 and 17 post-infection) was performed by $16 S$ rRNA gene sequencing. Additional file 2: Figure S2 shows estimates of the diversity of the microbiota, presented as plots of the Shannon index, Observed Species and Pielou index measure of adiversity. The a-diversity of the cecal microbiotas from chicks at 3 dpi was not neither affected by FFC treatment nor $S$. Enteritidis infection (Additional file 2: Figure S2a). However, a significantly decrease in alpha diversity was observed in FST group at $10 \mathrm{dpi}$ (Additional file 2: Figure S2b). And Additional file 2: Figure S2c shows that the Shannon and Pielou index of the cecal microbial communities are significantly increase in the FST group at $17 \mathrm{dpi}$. These results indicated that the a-diversity of gut microbiota from 
chicks are not significant affected by a single FFC treatment or Salmonella challenge. However, the infection of Salmonella after pretreatment with antibiotics significantly disturbed the alpha diversity of chicks.

The results of phylum and genus distributions of microbial composition are shown in Additional file 3: Figure S3 and Additional file 4: Figure S4, respectively. Firmicutes (71.40 - 99.62\%) dominated the chicks gut microbiota in the four groups at three different stages of infection (Additional file 3: Figure S3). At 3-, 10- and 17-days post infection, the FST group had the highest relative abundance of Proteobacteria (2.68\%, $1.30 \%$, and $1.32 \%$, respectively) compared with other three groups (Additional file 3: Figure S3). And at 17-days post infection, compared with the NT group (27.40\%), the FFC (7.76\%) significantly reduced the relative abundance of Bacteroidetes, and Salmonella infection (22.27\%) has less effect on Bacteroidetes. However, the infection of Salmonella after pretreatment with FFC almost limits the growth of Bacteroidetes $(0.01 \%)$ (Additional file 4: Figure S4). We further applied the LEfSe method to identify specifically abundant bacterial taxa among these groups (only those taxa that obtained a log linear discriminant analysis [LDA] scores $>3$ were ultimately considered). A cladogram from phylum to genus level abundance is shown in Fig. 6 . In total, 21, 21, and 28 differentially abundant bacterial taxa were identified at three different stages of infection, respectively (Fig. 6). In the non-treated chicks, LEfSe highlights the greater differential abundance of Lactobacillus at 3 and $10 \mathrm{dpi}$, and Bacteroides at $17 \mathrm{dpi}$. Notably, the relative abundance of Enterobacteriaceae was significantly higher in the FST group compared with other three groups at all three different points. However, the other taxa were changed irregularly at different times in different groups. Moreover, the relative abundance of these biomarkers was showed in Additional file 5: Figure S5, and consistent results are obtained. We also established taxonomic cladogram at 11 day ( $3 \mathrm{dpi})$, and the relative abundance of taxa node in each group was showed in the form of a pie chart (only those taxa that the relative abundance $>0.1 \%$ were ultimately considered) (Fig. 7a). Similarly, the abundance ratio of Lactobacillus in the control group was significantly higher than the other three groups. Additionally, the abundance ratio of Enterobacteriaceae in the FST group was dominated among these four groups. Furthermore, at the genus level, Salmonella was only found in the challenged groups, and the abundance ratio of Salmonella in the FFC pretreatment group was significantly higher than that in the unpretreated group (Fig. 7a). Additionally, we determined the cecal loads of these biomarkers and two intestinal protective bacteria by quantitative PCR (qPCR) (Fig. 7b). At 11 day (3 dpi), the FFC pre-treatment significantly reduced the densities of total bacteria, Lactobacillus, clostridium butyricum and faecalibacterium prausnitzii. Although single Salmonella infection had no effect on densities in the cecal contents, Salmonella infection after pretreatment with FFC group harbored much higher densities of Enterobacteriaceae, and lower densities of Lactobacillus, Bacteroides, clostridium butyricum and faecalibacterium prausnitzii than the control group. At 25 day (17 $\mathrm{dpi}$ ), the clostridium butyricum and faecalibacterium prausnitzii were present at equivalent densities in the cecal contents of four groups. However, the significant differences in the bacterial densities of total bacteria, Lactobacillus, Bacteroides and Enterobacteriaceae were still apparent between NT and FST group or ST and FST group (Fig. 7b). The Lactobacillus and Bacteroides are generally considered as the beneficial bacteria that provide protection for the gut, whereas the Enterobacteriaceae was known to be 
the potential pathogens of poultry and/or humans. These observations suggest that FFC exposure significantly decreased the abundance of Lactobacillus in chicks, and this inhibitory effect may provide a growth advantage for Enterobacteriaceae, especially Salmonella, in the gut of chicks.

The similarity of microbial communities ( $\beta$-diversity) was visualized through PCoA of Bray-Curtis distances. At 3 dpi. The PCoA plots showed that microbial communities from Salmonella or FFC treated chicks clearly separate from those of the non-treated chicks. The first axis of the PCoA explained $19.0 \%$ of the variation in bacterial diversity while the second axis explained $13.0 \%$ (Fig. 7C). The first axis can roughly distinguish the antibiotic pre-treated chicks and non-pretreated chicks, and second axis can roughly distinguish the Salmonella infected birds and non-infected birds. The PCoA at $10 \mathrm{dpi}$ showed that the microbiota composition was very similar between the NT and FT chicks, whereas the ST and FST group are still obviously distinguish from the NT group (Additional file 6: Figure S6a). Intriguingly, at 25 day (17 dpi), the PCoA demonstrated that both the microbiota composition of ST and FT group is tended to the NT group, whereas microbiota composition of FST group is still a striking divergence from the NT group (Additional file 6: Figure S6b). These findings suggest that a single FFC or Salmonella treatment can cause some changes in microbiota composition of chicks, and they would generally recover after two weeks. Whereas FFC pretreatment hindered the recovery from microbiota composition of chicks for Salmonella infection.

\section{Florfenicol administration alters the metabolic profiling}

We hypothesized that differences in key metabolites may be crucial to the effect of Salmonella colonization on chicks. Therefore, we conducted metabolomic analysis by LC-MS to determine the differential levels of metabolites on day 11 (3 dpi) in cecal contents of different groups. The principalcoordinate analysis (PCA) score plot showed that the metabolome of NT group and ST group was significantly separated among four groups, whereas there was no clear distinction in cecal metabolites between the FT and FST group (Fig. 8). For further analysis, orthogonal projections to latent structuresdiscriminate analysis (OPLS-DA) and permutation test plot of OPLS-DA was carried out to explore the differences between these groups. As shown in Additional file 7: Figure S7, the OPLS-DA showed that the cecal metabolites of the NT group were clearly distinguished from those of the FT group (Additional file 7: Figure S7a), ST group (Additional file 7: Figure S7c), and FST group (Additional file 7: Figure S7e). In addition, there was also a clear separation between the FST group and ST group in cecal metabolites (Additional file 7: Figure S7g).

From the OPLS-DA models, we identified 72 differential metabolites between NT and FT group, 42 differential metabolites between NT and ST group, 69 differential metabolites between NT and FST group, and 57 differential metabolites between FST and ST group according to the threshold (VIP > 1, and $p<0.05$; Welch's $t$ test). The significantly differential metabolites of these groups are shown in Supplementary Table 3. We next performed the pathway enrichment analysis based on these differential metabolites to comprehensively understand the effect of FFC on metabolism of chicks (Fig. 9). Linoleic acid metabolism, aminoacyl-tRNA biosynthesis, lysine biosynthesis, phenylalanine metabolism and 
lysine degradation were enriched after FFC treated (Fig. 9a). Arginine and proline metabolism, lysine biosynthesis, lysine degradation and D-glutamine and D-glutamate metabolism were enriched after Salmonella infected (Fig. 9b). Linoleic acid metabolism, aminoacyl-tRNA biosynthesis, lysine biosynthesis, butanoate metabolism and phenylalanine metabolism were enriched in Salmonella infection after FFC pretreatment group (Fig. 9c). Linoleic acid metabolism was enriched between the FST and ST group (Fig. 9d). The above results indicate that the linoleic acid metabolism is the most remarkable metabolism pathway in FFC treated group with or without Salmonella challenge. We next mapped the metabolic pathway of linoleic acid based on identified differential metabolites, and the relative amount (mean $\pm S D$ ) of these metabolites in four groups was also showed (Fig. 9e). The metabolites that affect the metabolic pathways of linoleic acid mainly include linoleic acid, 12,13-EpOME and 12,13-diHOME, and the relative amount of these metabolites in the FT and FST group was significantly higher than the NT and ST group. Notably, the relative levels of 12,13-EpOME and 12,13diHOME were significantly high in the FFC-pretreated group, but almost none in the non-pretreated group (Fig. 9e).

\section{Correlation between the differential gut microbiota and metabolites}

After finding marked differences in the content of metabolites as well as the microbial composition after FFC-pretreated, we analyzed whether there were any specific correlations between the microbial taxa and key metabolites. The Spearman correlation analysis revealed an association between four bacterial genera with nine discriminant metabolites in FFC-pretreated chicks (Fig. 10a). Enterobacteriaceae is a taxon with strong correlation, particularly with linoleic acid, 12,13-EpOME, 12,13-diHOME and L-tyrosine (positive correlations), while only L-ascorbic acid negatively correlated. Furthermore, the Clostridium positively correlated with L-palmitoylcarnitine, linoleic acid, 12,13-diHOME and L-tyrosine, while the taxon negatively correlated with L-ascorbic acid, anandamide and 4-pyridoxic acid. The Lactobacillus genus negatively correlated with L-palmitoylcarnitine, linoleic acid, 12,13-EpOME, 12,13-diHOME and L-tyrosine, and positively correlated with L-ascorbic acid. Lastly, a less strong positive correlation was detected between the Ruminococcus and 4-pyridoxic acid and gamma-aminobutyric acid. The CCA test showed that the Enterobacteriaceae was the most important bacterial factor influencing the linoleic acid metabolism (including linoleic acid, 12,13-EpOME, 12,13-diHOME) after FFC-pretreated (Fig. 10b). Additionally, the correlation network between differential bacterial taxa and metabolites is consists of 13 nodes and 22 edges. And the results also showed that the metabolic pathway of linoleic acid has a strong positive correlation with Enterobacteriaceae, while Lactobacillus negatively correlated (Fig. 10c).

Since linoleic acid can be produced by Lactobacillus into CLA [54], and in our study, there is a significant negative correlation between the linoleic acid and Lactobacillus. Therefore, we hypothesized that the nonFFC pretreated chicks (more abundance of Lactobacillus) may have more CLA contents. However, CLA is an isomer of linoleic acid, and the use of untargeted metabolomics detection cannot distinguish these substances, so we using the targeted LC-MS to detect these substances including linoleic acid, 9c,11tCLA, 10t,11c-CLA, 12,13-EpOME and 12,13-diHOME (Fig. 10d). In line with results of the metabolic profiling, the contents of linoleic acid, 12,13-EpOME and 12,13-diHOME were higher in the FFC-pretreated 
groups. Moreover, we confirmed more CLAs concentrations in the cecal contents of non-FFC pretreated chicks, and the 9c,11t-CLA level is significantly higher than the10t,11c-CLA (Fig. 10d). Furthermore, the Spearman correlation analysis showed a strong association between the abundance of lactobacillus and CLA concentrations (Additional file 8: Figure S8). Collectively, these findings hinted that 12,13-EpOME and 12,13-diHOME may be the key metabolites for prolonging gut colonization of Salmonella, whereas the CLA may limit the Salmonella growth during infection.

\section{Conjugated linoleic acid Attenuates, yet 12,13-diHOME promotes, the $S$. enteritidis Colonization}

To address if CLA and 12,13-diHOME affect the Salmonella colonization more directly, we preadministered these compounds to newly hatched chicks before infected with $S$. Enteritidis (Fig. 11a). By day 3 post-infection, the Salmonella loads in the caecum, spleen and liver were significantly reduced in the chicks pretreated with CLA, whereas those were significantly increased in the chicks pretreated with 12,13-diHOME (Fig. 11b). Consistent with the fecal Salmonella loads, the pre-treatment of CLA significantly reduced, whereas 12,13-diHOME significantly increased the enteropathy of chicks by $3 \mathrm{dpi}$ (Additional file 10: Figure S10). Furthermore, CLA-pretreated chicks also exhibited decreases intestinal permeability (serum D-lactate, DAO and LPS levels), and decreases pro-inflammatory levels (IL-1 $\beta$, IL-6, IL8 , TNF-a and IFN- $\gamma$ ), as well as a significant increase in IL-10 levels. Similarly, the 12,13-diHOMEpretreated chicks gets the opposite results (Fig. 12). We also compared the effect of these two metabolites on the expression of intestinal barrier function genes after Salmonella infection (Fig. 13). The results showed that the CLA significantly increased the expression of ZO-1 and Occludin, whereas the 12,13-diHOME significantly reduced the expression ZO-1, Occludin, Caludin1 and MUC2, and significantly increased the expression of IL-17A (Fig. 13). To evaluate whether orally administration CLA and 12,13diHOME reach to the gut lumen, we quantified the concentrations of these substances in the cecal contents, and observed a significant increase in cecal contents levels compared with non-treated chicks (Additional file 11: Figure S11). Together, these results demonstrate that pretreated CLA can attenuates, yet 12,13-diHOME promotes the Salmonella colonization in the gut of neonatal chicks.

\section{Discussion}

The antibiotics administration can perturb the gut bacterial community, resulting in weaken the gut colonization resistant to the pathogens $[22,45,53,55]$. Yet, the mechanisms that promotes Salmonella outgrowth after antibiotic pretreatment in chicks remain poorly described. In this study, we investigated the effect of antibiotic (FFC) pre-administration on the intestinal Salmonella colonization of chicks and its mechanism through microbiome and metabolomics. Similar to the reported papers [45,55], our results indicated that FFC can significantly increase the loads and prolongs gut colonization of $S$. Enteritidis. And the abundance of Salmonella also significantly increased in the endogenous organs (liver, spleen) exposed to FFC pretreatment. Salmonella depends on the two pathogenicity islands (SPI1and SPI2) to enter the intestine and adheres to the surfaces of intestinal epithelial cells to subsequently arrive in the subepithelial tissue via a series of invasive pathological pathways [56]. In the current study, we found that FFC-pretreatment could exacerbate Salmonella-induced morphology and intestinal barrier function injury 
and increased the intestinal barrier permeability. The animal assay revealed that FFC could directly decrease SIgA concentration, mucous layer density and increase the concentrations of serum DAO and LPS. And the real-time PCR demonstrated that FFC directly significantly decrease the expression of claudin 1, and increase the expression of IL-17A and IFN- $a$. The SIgA reflects the intestinal immunity state, and is able to stabilize intestinal colonization by symbiotic microorganisms and confer resistance to future invasion by exogenous pathogens [57-59]. Studies have shown that the gut microbiota is the most important source of immune microbial stimulation, use of antibiotics can disrupt the delicate ecosystem of the neonatal microbiome, which may cause an impaired stimulation of SIgA, and a low IgA response in turn lead to a reduced mucosal barrier function [60-62]. Furthermore, FFC can also aggravates Salmonella-induced inflammation in ileum, including upregulated the secretion of IL-1 $\beta$, IL-6, IL-8, INF- $y$, TNF- $a$, and decreased the IL-10 concentration. Previous study reported that the intestinal inflammation provides a growth advantage for Salmonella [53,63-65]. And our results showed that the FFC could directly increase the concentrations of these proinflammatory cytokines. Taken together, these findings imply that FFC pretreatment impaired intestinal immunity, increased intestinal permeability and inflammation, as well as aggravated Salmonella-induced intestinal barrier function damage, which promoted Salmonella colonization in neonatal chicks.

Based on the gut microbiota plays an important role in combating Salmonella invasion and maintaining intestinal immunity $[53,66]$, we explore the intestinal flora of neonatal chicks in different treated groups. The present study showed that the Firmicutes dominated the gut microbiota of neonatal chicks at day 11 and 18, and the mature microbial communities of chickens (at day 25) were dominated by Firmicutes and Bacteroidetes. This finding was consistent to previous studies $[67,68]$. However, FFC administration significantly decreased the abundance of Lactobacillus at day 11 and 18 ( 4 and 11 days post treated), and significantly decreased the Bacteroides at day 25 (18 days post treated). The Lactobacillus spp. are considered a probiotic and have been used in feed processing for decades because of their beneficial effects on immunity, growth, and intestinal colonization resistance of livestock [69-71]. For example, the Lactobacillus rhamnosus reduced the colonization of pathogenic Salmonella, Clostridium, and E. coli strains to the intestinal mucus of pig [72]. The Lactobacillus acidophilus can bind to cultured human intestinal cell lines and inhibit the cell invasion by enterovirulent bacteria including Salmonella Typhimurium [73]. And another study showed that the Lactobacillus plantarum exerts an antagonistic effect on pathogenic bacteria by increasing the content of SIgA [74]. In our study, the FFC treatment significantly decreased the abundance of Lactobacillus in chicks, which indicates that this genus may be the main target bacteria for FFC antimicrobial effects, and the similar observations have been found by others using FFC therapy on intestinal microbiota in chickens [75]. And this reduction may be responsible for the promotion of Salmonella colonization after FFC pre-treatment. Bacteroidetes is the dominant phylum in the mature microbiota of chickens [76], and also have some inhibit effects on the gut colonization of Salmonella. Miki et al. find that Bacteroides spp. can accelerate Salmonella Typhimurium elimination from the intestinal lumen of mice by producing vitamin B6 [45]. And another study demonstrates that the Bacteroides species confer colonization resistance to Salmonella Typhimurium infection by producing the propionate, which directly limits Salmonella growth by disrupting intracellular 
$\mathrm{pH}$ homeostasis [77]. And our results showed that at day 25, FFC pretreatment significantly reduced the abundance of Bacteroidetes and had more Salmonella loads in the cecum compared with the nonpretreated group, suggesting that FFC may have delayed the maturation of chicken intestinal flora and hinder clearance of Salmonella. Furthermore, the Salmonella infection after FFC pre-treated chicks had the had the highest relative abundance of Proteobacteria, which was known to be the potential pathogens of poultry and/or humans. The recent study showed that the florfenicol preventive treatment of calves showed a 10-fold increase in facultative anaerobic Escherichia spp, which a signature of imbalanced microbiota [78]. And Sáenz et al. oral administration of florfenicol to the fish observed a shift in the gut microbiome towards well-known putative pathogens such as Salmonella, Plesiomonas, and Citrobacter [79]. Combined with our results, it was showed that the FFC administration could changes the overall structure of the gut microbiota and promotes the growth of Proteobacteria, especially Salmonella. Moreover, although the microbial community of chicken is complex and relative stable, and the restoration of the microbiota after antibiotics withdrawal could be expected [80,81], our results indicate that using antibiotics at an early age of chickens could have a profound effect on microbial composition (Additional file 5: Figure S5). And the study also demonstrated that the maturation of intestinal microbiota was significantly retarded and eventually delayed by antibiotics intervention at early ages of chicks [82].

We next use metabolomics to analyze how may FFC affect Salmonella gut colonization. Our data reported here suggest that the linoleic acid metabolism is the most remarkable metabolism pathway for FFC pre-administration. And we identified that the linoleic acid, 12,13-EpOME and 12,13-diHOME are the most important substances affecting linoleic acid metabolic pathway, which contents of these metabolites are significantly higher after FFC pre-treatment. It's worth noting that the concentrations of 12,13-EpOME and 12,13-diHOME were significantly high in the FFC-pretreated group, but almost none in the non-pretreated group. Linoleic acid is firstly metabolized to 12,13-EpOME by cytochrome P450 (CYP) epoxygenases, and followed by hydrolysis catalyzed by soluble epoxide hydrolases (sEHs) to form the diols 12,13-diHOME [83]. They have multiple pathological features, such as decreasing post-ischemic cardiac recovery, participating in the vascular cognitive impairment, increasing skeletal muscle fatty acid uptake and impeding immune tolerance in asthma child [31, 84-86]. And the 12,13-diHOME produced by sEH hydrolysis of 12,13-EpOME showed stronger cytotoxicity $[83,87]$. Our analysis of metabolic enzymes in the pathway found that FFC can significantly increase the expression of CYP1A2, whereas had no significant effect on SEH (Additional file 9: Figure S9). Besides SEH produced by the liver, a variety of gut bacteria can also produce [31]. Correlation analysis results showed that the concentration of 12,13diHOME was significantly positively correlated with Enterobacteriaceae and Clostridium, so we suspected that the $\mathrm{sEH}$ may be produced by these bacteria in the gut. A recent study showed that the sEH and sEHproduced lipid metabolites induces intestinal barrier dysfunction, bacterial translocation and colonic inflammation in mice [88]. Therefore, we suggest that 12,13-diHOME may promotes the intestinal colonization of Salmonella. Then, we pretreated with 12,13-diHOME for neonatal chicks and found that it significantly increased Salmonella colonization. Our results also showed that the 12,13-diHOME pretreatment significantly increase the Salmonella-induced expression of intestinal proinflammatory 
cytokines, exacerbate morphology and intestinal barrier function injury and increased the intestinal barrier permeability. The intestinal inflammation, particularly that due to proinflammatory cytokines, can disrupt barrier function and lead to intestinal permeability, and promote the pathogens colonization $[63,89,90]$. Previous studies showed that the diHOMEs has the pro-inflammatory effect on vascular endothelial cells [91], lung [31] and peripheral nervous tissue [92], and our study indicate that the 12,13-diHOME also has a pro-inflammatory effect on intestinal epithelial cells. Moreover, the diHOMEs have also been shown to disrupt mitochondrial function, eliciting the mitochondrial permeability transition and causing cellular apoptosis [93, 94], and this may be why 12,13-diHOME exacerbates intestinal barrier damage. Therefore, it suggests that 12,13-diHOME could contribute to Salmonella colonization in the intestinal of chicks, in partly by promoting intestinal inflammation and destroying the intestinal barrier function.

CLA is the second factor affecting the Salmonella gut colonization after FFC pre-administration. Our correlation analysis combined with targeted metabolomic found that the Lactobacillus and CLA showed a significant positive correlation, and FFC pretreatment both significantly reduced the abundance of Lactobacillus and CLA in the gut lumen. CLA can be formed from linoleic acid by Lactobacillus and can inhibit the growth of the pathogenic bacteria [95]. Therefore, we think the CLA may be another factor that affects Salmonella colonization after FFC pretreatment. We pretreated with CLA for neonatal chicks and found that it effectively reduced Salmonella colonization, accompanied by the increased the expression of tight junction proteins (ZO-1 and occludin), alleviated the Salmonella-induced intestinal inflammation, and intestinal barrier injury. And we believe that CLA reduces the Salmonella intestinal colonization including several aspects. Firstly, CLA treatments could significantly upregulated the concentration of tight junction proteins (ZO-1, occludin, E-cadherin 1 and claudin-3) and ameliorated epithelial apoptosis [96-98], which protect intestinal from the impairments caused by Salmonella infection. Secondly, CLA can modulate the gut inflammation including attenuated the expression of proinflammatory cytokines (TNF- $\alpha$, INF-y, IL-1 $\beta$, and IL- 6 and IL-17) while upregulating the level of the anti-inflammatory cytokine IL10 [96-101]. The anti-inflammatory effect of CLA could reduce the gut colonization of Salmonella. Finally, the studies also showed that the CLA can directly inhibit the growth of the pathogenic bacteria including Salmonella. Byeon et al. showed that the CLA can against the growth of a variety of food-borne pathogens, and the $1.8 \mathrm{mM}$ CLA could completely inhibited the growth of Salmonella Typhimurium [95]. Peng et al. indicated that the CLA produced by Lactobacillus can competitively excluded Salmonella in a mixed-culture condition [102]. And Tabashsum et at. also showed that the CLA produced by Lactobacillus inhibited the growth and survival of Salmonella by altering the relative expression of genes related to Salmonella virulence [103]. Thus, our results suggest that CLA can maintain intestinal integrity, reduce intestinal inflammation, and inhibit Salmonella growth to effectively reduce the gut colonization of Salmonella in chicks. In view of the fact that CLA can be produced by Lactobacillus, and our research shows that FFC treatment dramatically reduce the content of Lactobacillus and CLA. Therefore, FFC may reduce the production of CLA by inhibiting the Lactobacillus growth, thereby reducing the colonization resistance of neonatal chicks to Salmonella infection.

\section{Conclusion}


Taken together, this study indicates that FFC pre-treatment significantly increases gut susceptibility to $S$. Enteritidis, and significantly increases the Salmonella-induced inflammatory responses and intestinal barrier damage in neonatal chicks. The metagenomic and metabonomic analysis revealed that FFC pretreatment significantly reduced the content of Lactobacillus, and significantly affected the linoleic acid metabolism pathway, including significantly reducing the levels of CLA, and significantly increasing the abundance of 12,13-EpOME and 12,13-diHOME. And we found that the CLA can maintain intestinal integrity, reduce intestinal inflammation, and directly inhibit Salmonella growth to effectively reduce the Salmonella colonization in chicks. Whereas the 12,13-diHOME though promoting intestinal inflammation and destroying the intestinal barrier function to support the Salmonella colonization. These findings suggest that the decreased levels of Lactobacillus and CLA, and elevated cecal 12,13-diHOME concentrations in FFC pre-treated neonatal chicks might be an intestinal health-impairing attribute and may contribute to Salmonella colonization.

\section{Declarations}

\section{Funding}

This work was financially supported by the General Program of National Natural Science Foundation of China (grant No. 31830098 and 3177131163), China Agriculture Research System National System for Layer Production Technology (grant No. CARS-40-K14), and the National Key R\&D Program of China (grant No. 2016YFD0501608).

\section{Availability of data and materials}

The raw sequence data obtained in this study has been deposited in the Sequence Read Archive (SRA) database of the National Center for Biotechnology Information (NCBI) with an access number of SRP277009.

\section{Authors' contributions}

HW and XM conceived the study and designed the experiments. $X M, B M, X Z, L Z, Y G$ and $C Z$ collected the samples and performed experiments. $H W, X M, B M$ and $X Z$ analyzed the data. $A Z, C L, Y T$ and $X Y$ provide suggestions and help checking. $\mathrm{HW}, \mathrm{XM}$ and $\mathrm{BM}$ wrote the manuscript. $\mathrm{AZ}, \mathrm{CL}, \mathrm{YT}, \mathrm{XY}, \mathrm{YG}$ and $\mathrm{CZ}$ help revise the manuscript. The final manuscript was read and approved by all authors.

\section{Ethics approval}

All experiments in this study were reviewed and approved by the Institutional Animal Care and Use Committee at the Sichuan University.

\section{Competing interests}

The authors declare that they have no competing interests. 


\section{Publisher's Note}

Springer Nature remains neutral with regard to jurisdictional claims in published maps and institutional affiliations.

\section{Author details}

${ }^{1}$ Key Laboratory of Bio-Resource and Eco-Environment of Ministry of Education, College of Life Sciences, Sichuan University, Chengdu 610065, Sichuan, P.R. China.

${ }^{2}$ Animal Disease Prevention and Food Safety Key Laboratory of Sichuan Province, Chengdu, Sichuan, P.R. China.

${ }^{3}$ Department of Biological Engineering, Sichuan Water Conservancy Vocational College, Chengdu, Sichuan, P.R. China.

\section{References}

1. U. D: World population projected to reach 9.7 billion by 2050. 2015.

2. Scanes CG. The global importance of poultry. Poult Sci. 2007;86:1057-8.

3. Grace D, Mutua F, Ochungo P, Kruska R, Jones K, Brierley L, Lapar L, Said M, Herrero M, Phuc P. Mapping of poverty and likely zoonoses hotspots. In: Zoonoses Project 4 . Report to the UK Department for International Development. Nairobi: International Livestock Research Institute; 2012.

4. Dunkley KD, Callaway TR, Chalova VI, McReynolds JL, Hume ME, Dunkley CS, Kubena LF, Nisbet DJ, Ricke SC. Foodborne Salmonella ecology in the avian gastrointestinal tract. Anaerobe. 2009;15:2635 .

5. Khan SB, Khan MA, Ahmad I, Ur Rehman T, Ullah S, Dad R, Sultan A, Memon AM. Phentotypic, gentotypic antimicrobial resistance and pathogenicity of Salmonella enterica serovars Typimurium and Enteriditis in poultry and poultry products. Microb Pathog. 2019;129:118-24.

6. Kirk MD, Pires SM, Black RE, Caipo M, Crump JA, Devleesschauwer B, Dopfer D, Fazil A, FischerWalker CL, Hald T. World Health Organization Estimates of the Global and Regional Disease Burden of 22 Foodborne Bacterial, Protozoal, and Viral Diseases, 2010: A Data Synthesis. PLoS Med. 2015;12:e1001921.

7. Martelli F, Davies RH. Salmonella serovars isolated from table eggs: An overview. Food Res Int. 2012;45:745-54.

8. European Food Safety A. European Centre for Disease P, Control: The European Union One Health 2018 Zoonoses Report. EFSA J. 2019;17:e05926.

9. Dewey-Mattia D, Manikonda K, Hall AJ, Wise ME, Crowe SJ. Surveillance for Foodborne Disease Outbreaks - United States, 2009-2015. MMWR Surveill Summ. 2018;67:1-11.

10. Velasquez-Manoff M. Gut microbiome: the peacekeepers. Nature. 2015;518:3-11. 
11. Baumler AJ, Sperandio V. Interactions between the microbiota and pathogenic bacteria in the gut. Nature. 2016;535:85-93.

12. Sassone-Corsi M, Raffatellu M. No Vacancy: How Beneficial Microbes Cooperate with Immunity To Provide Colonization Resistance to Pathogens. J Immunol. 2015;194:4081-7.

13. Stanley D, Hughes RJ, Moore RJ. Microbiota of the chicken gastrointestinal tract: influence on health, productivity and disease. Appl Microbiol Biotechnol. 2014;98:4301-10.

14. Tang Y, Underwood A, Gielbert A, Woodward MJ, Petrovska L. Metaproteomics analysis reveals the adaptation process for the chicken gut microbiota. Appl Environ Microbiol. 2014;80:478-85.

15. Modi SR, Collins JJ, Relman DA. Antibiotics and the gut microbiota. J Clin Invest. 2014;124:4212-8.

16. Xiong W, Wang Y, Sun Y, Ma L, Zeng Q, Jiang X, Li A, Zeng Z, Zhang T. Antibiotic-mediated changes in the fecal microbiome of broiler chickens define the incidence of antibiotic resistance genes. Microbiome. 2018;6:34.

17. Zhang QQ, Ying GG, Pan CG, Liu YS, Zhao JL. Comprehensive evaluation of antibiotics emission and fate in the river basins of China. source analysis, multimedia modeling, and linkage to bacterial resistance. Environ Sci Technol. 2015;49:6772-82.

18. Van Boeckel TP, Brower C, Gilbert M, Grenfell BT, Levin SA, Robinson TP, Teillant A, Laxminarayan R. Global trends in antimicrobial use in food animals. Proc Natl Acad Sci U S A. 2015;112:5649-54.

19. Molbak K. Human health consequences of antimicrobial drug-resistant Salmonella and other foodborne pathogens. Clin Infect Dis. 2005;41:1613-20.

20. Stecher B, Hardt WD. The role of microbiota in infectious disease. Trends Microbiol. 2008;16:107-14.

21. Kim HB, Borewicz K, White BA, Singer RS, Sreevatsan S, Tu ZJ, Isaacson RE. Longitudinal investigation of the age-related bacterial diversity in the feces of commercial pigs. Vet Microbiol. 2011;153:124-33.

22. Barthel M, Hapfelmeier S, Quintanilla-Martinez L, Kremer M, Rohde M, Hogardt M, Pfeffer K, Russmann H, Hardt WD. Pretreatment of mice with streptomycin provides a Salmonella enterica serovar typhimurium colitis model that allows analysis of both pathogen and host. Infect Immun. 2003;71:2839-58.

23. Lawley TD, Bouley DA, Hoy YE, Gerke C, Relman DA, Monack DM. Host transmission of Salmonella enterica serovar Typhimurium is controlled by virulence factors and indigenous intestinal microbiota. Infect Immun. 2008;76:403-16.

24. Sadler WW, Brownell JR, Fanelli MJ. Influence of age and inoculum level on shed pattern of Salmonella typhimurium in chickens. Avian Dis. 1969;13:793-803.

25. Crhanova M, Hradecka H, Faldynova M, Matulova M, Havlickova H, Sisak F, Rychlik I. Immune response of chicken gut to natural colonization by gut microflora and to Salmonella enterica serovar enteritidis infection. Infect Immun. 2011;79:2755-63.

26. Nurmi E, Rantala M. New aspects of Salmonella infection in broiler production. Nature. 1973;241:210-1. 
27. Goren E, de Jong WA, Doornenbal P, Bolder NM, Mulder RW, Jansen A. Reduction of salmonella infection of broilers by spray application of intestinal microflora: a longitudinal study. Vet Q. 1988;10:249-55.

28. Juricova H, Videnska P, Lukac M, Faldynova M, Babak V, Havlickova H, Sisak F, Rychlik I. Influence of Salmonella enterica serovar enteritidis infection on the development of the cecum microbiota in newly hatched chicks. Appl Environ Microbiol. 2013;79:745-7.

29. Hosoi Y, Asai T, Koike R, Tsuyuki M, Sugiura K. Sales of veterinary antimicrobial agents for therapeutic use in food-producing animal species in Japan between 2005 and 2010. Rev Sci Tech. 2014;33:1007-15.

30. Philippe N, Alcaraz JP, Coursange E, Geiselmann J, Schneider D. Improvement of pCVD442, a suicide plasmid for gene allele exchange in bacteria. Plasmid. 2004;51:246-55.

31. Levan SR, Stamnes KA, Lin DL, Panzer AR, Fukui E, McCauley K, Fujimura KE, McKean M, Ownby DR, Zoratti EM. Elevated faecal 12,13-diHOME concentration in neonates at high risk for asthma is produced by gut bacteria and impedes immune tolerance. Nat Microbiol. 2019;4:1851-61.

32. de Souza M, Baptista AAS, Valdiviezo MJJ, Justino L, Menck-Costa MF, Ferraz CR, da Gloria EM, Verri WA Jr, Bracarense A. Lactobacillus spp. Reduces morphological changes and oxidative stress induced by deoxynivalenol on the intestine and liver of broilers. Toxicon. 2020.

33. Xu S, Wang D, Zhang P, Lin Y, Fang Z, Che L, Wu D. Oral administration of Lactococcus lactisexpressed recombinant porcine epidermal growth factor stimulates the development and promotes the health of small intestines in early-weaned piglets. J Appl Microbiol. 2015;119:225-35.

34. Evans CC, LePard KJ, Kwak JW, Stancukas MC, Laskowski S, Dougherty J, Moulton L, Glawe A, Wang $Y$, Leone V. Exercise prevents weight gain and alters the gut microbiota in a mouse model of high fat diet-induced obesity. PLoS One. 2014;9:e92193.

35. Gill SR, Pop M, Deboy RT, Eckburg PB, Turnbaugh PJ, Samuel BS, Gordon JI, Relman DA, FraserLiggett CM, Nelson KE. Metagenomic analysis of the human distal gut microbiome. Science. 2006;312:1355-9.

36. Chen $\mathrm{H}$, Jiang W. Application of high-throughput sequencing in understanding human oral microbiome related with health and disease. Front Microbiol. 2014;5:508.

37. Magoc T, Salzberg SL. FLASH: fast length adjustment of short reads to improve genome assemblies. Bioinformatics. 2011;27:2957-63.

38. Edgar RC. Search and clustering orders of magnitude faster than BLAST. Bioinformatics. 2010;26:2460-1.

39. DeSantis TZ, Hugenholtz P, Larsen N, Rojas M, Brodie EL, Keller K, Huber T, Dalevi D, Hu P, Andersen GL. Greengenes, a chimera-checked 16S rRNA gene database and workbench compatible with ARB. Appl Environ Microbiol. 2006;72:5069-72.

40. Altschul SF, Madden TL, Schaffer AA, Zhang J, Zhang Z, Miller W, Lipman DJ. Gapped BLAST and PSI-BLAST: a new generation of protein database search programs. Nucleic Acids Res. 1997;25:3389-402. 
41. Caporaso JG, Kuczynski J, Stombaugh J, Bittinger K, Bushman FD, Costello EK, Fierer N, Pena AG, Goodrich JK, Gordon JI. QIIME allows analysis of high-throughput community sequencing data. Nat Methods. 2010;7:335-6.

42. Huson DH, Mitra S, Ruscheweyh HJ, Weber N, Schuster SC. Integrative analysis of environmental sequences using MEGAN4. Genome Res. 2011;21:1552-60.

43. Asnicar F, Weingart G, Tickle TL, Huttenhower C, Segata N. Compact graphical representation of phylogenetic data and metadata with GraPhIAn. PeerJ. 2015;3:e1029.

44. Segata N, Izard J, Waldron L, Gevers D, Miropolsky L, Garrett WS, Huttenhower C. Metagenomic biomarker discovery and explanation. Genome Biol. 2011;12:R60.

45. Miki T, Goto R, Fujimoto M, Okada N, Hardt WD. The Bactericidal Lectin Reglllbeta Prolongs Gut Colonization and Enteropathy in the Streptomycin Mouse Model for Salmonella Diarrhea. Cell Host Microbe. 2017;21:195-207.

46. Bartosch S, Fite A, Macfarlane GT, McMurdo MET. Characterization of bacterial communities in feces from healthy elderly volunteers and hospitalized elderly patients by using real-time PCR and effects of antibiotic treatment on the fecal microbiota. Appl Environ Microb. 2004;70:3575-81.

47. Matsuda K, Tsuji H, Asahara T, Kado Y, Nomoto K. Sensitive quantitative detection of commensal bacteria by rRNA-targeted reverse transcription-PCR. Appl Environ Microb. 2007;73:32-9.

48. Bergstrom A, Licht TR, Wilcks A, Andersen JB, Schmidt LR, Gronlund HA, Vigsnaes LK, Michaelsen KF, Bahl MI. Introducing GUt low-density array (GULDA): a validated approach for qPCR-based intestinal microbial community analysis. FEMS Microbiol Lett. 2012;337:38-47.

49. Wang P, Xu J, Wang Y, Cao X. An interferon-independent IncRNA promotes viral replication by modulating cellular metabolism. Science. 2017;358:1051-5.

50. Xia J, Sinelnikov IV, Han B, Wishart DS. MetaboAnalyst 3.0-making metabolomics more meaningful. Nucleic Acids Res. 2015;43:W251-7.

51. Li HX, Liu XL, Chen FY, Zuo KJ, Wu C, Yan YM, Chen WG, Lin WC, Xie QM. Avian Influenza Virus Subtype H9N2 Affects Intestinal Microbiota, Barrier Structure Injury, and Inflammatory Intestinal Disease in the Chicken Ileum. Viruses-Basel. 2018;10.

52. Sun LH, Zhang NY, Zhu MK, Zhao L, Zhou JC, Qi DS. Prevention of Aflatoxin B1 Hepatoxicity by Dietary Selenium Is Associated with Inhibition of Cytochrome P450 Isozymes and Up-Regulation of 6 Selenoprotein Genes in Chick Liver. J Nutr. 2015;146:655-61.

53. Stecher B, Robbiani R, Walker AW, Westendorf AM, Barthel M, Kremer M, Chaffron S, Macpherson AJ, Buer J, Parkhill J. Salmonella enterica serovar typhimurium exploits inflammation to compete with the intestinal microbiota. PLoS Biol. 2007;5:2177-89.

54. Ogawa J, Kishino S, Ando A, Sugimoto S, Mihara K, Shimizu S. Production of conjugated fatty acids by lactic acid bacteria. J Biosci Bioeng. 2005;100:355-64.

55. Kumar S, Chen C, Indugu N, Werlang GO, Singh M, Kim WK, Thippareddi H. Effect of antibiotic withdrawal in feed on chicken gut microbial dynamics, immunity, growth performance and prevalence of foodborne pathogens. PLoS One. 2018;13:e0192450. 
56. Coburn B, GrassI GA, Finlay BB. Salmonella, the host and disease: a brief review. Immunol Cell Biol. 2007;85:112-8.

57. Luo Q, Cui H, Peng X, Fang J, Zuo Z, Deng J, Liu J, Deng Y. Intestinal IgA(+) cell numbers as well as $\lg A$, IgG, and IgM contents correlate with mucosal humoral immunity of broilers during supplementation with high fluorine in the diets. Biol Trace Elem Res. 2013;154:62-72.

58. Mantis NJ, Rol N, Corthesy B. Secretory IgA's complex roles in immunity and mucosal homeostasis in the gut. Mucosal Immunol. 2011;4:603-11.

59. Donaldson GP, Ladinsky MS, Yu KB, Sanders JG, Yoo BB, Chou WC, Conner ME, Earl AM, Knight R, Bjorkman PJ. Gut microbiota utilize immunoglobulin A for mucosal colonization. Science. 2018;360:795-800.

60. Tamburini S, Shen N, Wu HC, Clemente JC. The microbiome in early life: implications for health outcomes. Nat Med. 2016;22:713-22.

61. Ximenez C, Torres J. Development of Microbiota in Infants and its Role in Maturation of Gut Mucosa and Immune System. Arch Med Res. 2017;48:666-80.

62. Nogacka AM, Salazar N, Arboleya S, Suarez M, Fernandez N, Solis G, de L Reyes-Gavilan, Gueimonde CG. M. Early microbiota, antibiotics and health. Cell Mol Life Sci. 2018;75:83-91.

63. Thiennimitr P, Winter SE, Winter MG, Xavier MN, Tolstikov V, Huseby DL, Sterzenbach T, Tsolis RM, Roth JR, Baumler AJ. Intestinal inflammation allows Salmonella to use ethanolamine to compete with the microbiota. Proc Natl Acad Sci U S A. 2011;108:17480-5.

64. Winter SE, Thiennimitr P, Winter MG, Butler BP, Huseby DL, Crawford RW, Russell JM, Bevins CL, Adams LG, Tsolis RM. Gut inflammation provides a respiratory electron acceptor for Salmonella. Nature. 2010;467:426-9.

65. Sekirov I, Gill N, Jogova M, Tam N, Robertson M, de Llanos R, Li Y, Finlay BB. Salmonella SPI-1mediated neutrophil recruitment during enteric colitis is associated with reduction and alteration in intestinal microbiota. Gut Microbes. 2010;1:30-41.

66. Hooper LV, Gordon JI. Commensal host-bacterial relationships in the gut. Science. 2001;292:111518.

67. Litvak Y, Mon KKZ, Nguyen H, Chanthavixay G, Liou M, Velazquez EM, Kutter L, Alcantara MA, Byndloss MX, Tiffany CR. Commensal Enterobacteriaceae Protect against Salmonella Colonization through Oxygen Competition. Cell Host Microbe. 2019;25:128-39 e125.

68. Pandit RJ, Hinsu AT, Patel NV, Koringa PG, Jakhesara SJ, Thakkar JR, Shah TM, Limon G, Psifidi A, Guitian J. Microbial diversity and community composition of caecal microbiota in commercial and indigenous Indian chickens determined using 16 s rDNA amplicon sequencing. Microbiome. 2018;6:115.

69. Patterson JA, Burkholder KM. Application of prebiotics and probiotics in poultry production. Poult Sci. 2003;82:627-31.

70. Abudabos AM, Al-Batshan HA, Murshed MA. Effects of prebiotics and probiotics on the performance and bacterial colonization of broiler chickens. S Afr J Anim Sci. 2015;45:419-28. 
71. Surendran Nair M, Amalaradjou MA, Venkitanarayanan K. Antivirulence Properties of Probiotics in Combating Microbial Pathogenesis. Adv Appl Microbiol. 2017;98:1-29.

72. Collado MC, Grzeskowiak L, Salminen S. Probiotic strains and their combination inhibit in vitro adhesion of pathogens to pig intestinal mucosa. Curr Microbiol. 2007;55:260-5.

73. Bernet MF, Brassart D, Neeser JR, Servin AL. Lactobacillus acidophilus LA 1 binds to cultured human intestinal cell lines and inhibits cell attachment and cell invasion by enterovirulent bacteria. Gut. 1994;35:483-9.

74. Wang L, Zhang J, Guo Z, Kwok L, Ma C, Zhang W, Lv Q, Huang W, Zhang H. Effect of oral consumption of probiotic Lactobacillus planatarum P-8 on fecal microbiota, SIgA, SCFAs, and TBAs of adults of different ages. Nutrition. 2014.30:776-83 e771.

75. Chen Y, Sun J, Liao XP, Shao Y, Li L, Fang LX, Liu YH. Impact of enrofloxacin and florfenicol therapy on the spread of OqxAB gene and intestinal microbiota in chickens. Vet Microbiol. 2016;192:1-9.

76. Dong XY, Azzam MMM, Zou XT. Effects of dietary threonine supplementation on intestinal barrier function and gut microbiota of laying hens. Poult Sci. 2017;96:3654-63.

77. Jacobson A, Lam L, Rajendram M, Tamburini F, Honeycutt J, Pham T, Van Treuren W, Pruss K, Stabler SR, Lugo K, et al. A Gut Commensal-Produced Metabolite Mediates Colonization Resistance to Salmonella Infection. Cell Host Microbe. 2018;24:296-307 e297.

78. Dobrzanska DA, Lamaudiere MTF, Rollason J, Acton L, Duncan M, Compton S, Simms J, Weedall GD, Morozov IY. Preventive antibiotic treatment of calves: emergence of dysbiosis causing propagation of obese state-associated and mobile multidrug resistance-carrying bacteria. Microb Biotechnol. 2020;13:669-82.

79. Saenz JS, Marques TV, Barone RSC, Cyrino JEP, Kublik S, Nesme J, Schloter M, Rath S, Vestergaard G. Oral administration of antibiotics increased the potential mobility of bacterial resistance genes in the gut of the fish Piaractus mesopotamicus. Microbiome. 2019;7:24.

80. Robinson CJ, Bohannan BJ, Young VB. From structure to function: the ecology of host-associated microbial communities. Microbiol Mol Biol Rev. 2010;74:453-76.

81. Macfarlane S. Antibiotic treatments and microbes in the gut. Environ Microbiol. 2014;16:919-24.

82. Gao P, Ma C, Sun Z, Wang L, Huang S, Su X, Xu J, Zhang H. Feed-additive probiotics accelerate yet antibiotics delay intestinal microbiota maturation in broiler chicken. Microbiome. 2017;5:91.

83. Moghaddam MF, Grant DF, Cheek JM, Greene JF, Williamson KC, Hammock BD. Bioactivation of leukotoxins to their toxic diols by epoxide hydrolase. Nat Med. 1997;3:562-6.

84. Bannehr M, Lohr L, Gelep J, Haverkamp W, Schunck WH, Gollasch M, Wutzler A. Linoleic Acid Metabolite DiHOME Decreases Post-ischemic Cardiac Recovery in Murine Hearts. Cardiovasc Toxicol. 2019;19:365-71.

85. Yu D, Hennebelle M, Sahlas DJ, Ramirez J, Gao F, Masellis M, Cogo-Moreira H, Swartz RH, Herrmann N, Chan PC. Soluble Epoxide Hydrolase-Derived Linoleic Acid Oxylipins in Serum Are Associated with Periventricular White Matter Hyperintensities and Vascular Cognitive Impairment. TransI Stroke Res. 2019;10:522-33. 
86. Stanford KI, Lynes MD, Takahashi H, Baer LA, Arts PJ, May FJ, Lehnig AC, Middelbeek RJW, Richard JJ, So K, et al: 12,13-diHOME. An Exercise-Induced Lipokine that Increases Skeletal Muscle Fatty Acid Uptake. Cell Metab. 2018;27:1111-20 e1113.

87. Newman JW, Morisseau C, Hammock BD. Epoxide hydrolases: their roles and interactions with lipid metabolism. Prog Lipid Res. 2005;44:1-51.

88. Wang Y, Yang J, Wang W, Sanidad KZ, Cinelli MA, Wan D, Hwang SH, Kim D, Lee KSS, Xiao H. Soluble epoxide hydrolase is an endogenous regulator of obesity-induced intestinal barrier dysfunction and bacterial translocation. Proc Natl Acad Sci U S A. 2020;117:8431-6.

89. Bruewer M, Luegering A, Kucharzik T, Parkos CA, Madara JL, Hopkins AM, Nusrat A. Proinflammatory cytokines disrupt epithelial barrier function by apoptosis-independent mechanisms. J Immunol. 2003;171:6164-72.

90. Turner JR. Intestinal mucosal barrier function in health and disease. Nat Rev Immunol. 2009;9:799809.

91. Slim R, Hammock BD, Toborek M, Robertson LW, Newman JW, Morisseau CH, Watkins BA, Saraswathi V, Hennig B. The role of methyl-linoleic acid epoxide and diol metabolites in the amplified toxicity of linoleic acid and polychlorinated biphenyls to vascular endothelial cells. Toxicol Appl Pharmacol. 2001;171:184-93.

92. Zimmer B, Angioni C, Osthues T, Toewe A, Thomas D, Pierre SC, Geisslinger G, Scholich K, Sisignano M. The oxidized linoleic acid metabolite 12,13-DiHOME mediates thermal hyperalgesia during inflammatory pain. Biochim Biophys Acta Mol Cell Biol Lipids. 2018;1863:669-78.

93. Moran JH, Mon T, Hendrickson TL, Mitchell LA, Grant DF. Defining mechanisms of toxicity for linoleic acid monoepoxides and diols in Sf-21 cells. Chem Res Toxicol. 2001;14:431-7.

94. Sisemore MF, Zheng J, Yang JC, Thompson DA, Plopper CG, Cortopassi GA, Hammock BD. Cellular characterization of leukotoxin diol-induced mitochondrial dysfunction. Arch Biochem Biophys. 2001;392:32-7.

95. Byeon JI, Song HS, Oh TW, Kim YS, Choi BD, Kim HC, Kim JO, Shim KH, Ha YL. Growth inhibition of foodborne and pathogenic bacteria by conjugated linoleic acid. J Agric Food Chem. 2009;57:316472.

96. Ren Q, Yang B, Zhang H, Ross RP, Stanton C, Chen H, Chen W. c9, t11, c15-CLNA and t9, t11, c15CLNA from Lactobacillus plantarum ZS2058 Ameliorate Dextran Sodium Sulfate-Induced Colitis in Mice. J Agric Food Chem. 2020;68:3758-69.

97. Peng M, Tabashsum Z, Patel P, Bernhardt C, Biswas C, Meng J, Biswas D. Prevention of enteric bacterial infections and modulation of gut microbiota with conjugated linoleic acids producing Lactobacillus in mice. Gut Microbes. 2020;11:433-52.

98. Chen Y, Yang B, Ross RP, Jin Y, Stanton C, Zhao J, Zhang H, Chen W. Orally Administered CLA Ameliorates DSS-Induced Colitis in Mice via Intestinal Barrier Improvement, Oxidative Stress Reduction, and Inflammatory Cytokine and Gut Microbiota Modulation. J Agric Food Chem. 2019;67:13282-98. 
99. Moreira TG, Gomes-Santos AC, Horta LS, Goncalves MC, Santiago AF, Lauar JG, Dos Reis DS, CastroJunior AB, Lemos L, Guimaraes M. Consumption of conjugated linoleic acid (CLA)-supplemented diet during colitis development ameliorates gut inflammation without causing steatosis in mice. J Nutr Biochem. 2018;57:238-45.

100. Foote MR, Giesy SL, Bernal-Santos G, Bauman DE, Boisclair YR. t10,c12-CLA decreases adiposity in peripubertal mice without dose-related detrimental effects on mammary development, inflammation status, and metabolism. Am J Physiol Regul Integr Comp Physiol. 2010;299:R1521-8.

101. Bassaganya-Riera J, Hontecillas R, Horne WT, Sandridge M, Herfarth HH, Bloomfeld R, Isaacs KL. Conjugated linoleic acid modulates immune responses in patients with mild to moderately active Crohn's disease. Clin Nutr. 2012;31:721-7.

102. Peng M, Tabashsum Z, Patel P, Bernhardt C, Biswas D. Linoleic Acids Overproducing Lactobacillus casei Limits Growth, Survival, and Virulence of Salmonella Typhimurium and Enterohaemorrhagic Escherichia coli. Front Microbiol. 2018;9:2663.

103. Tabashsum Z, Peng M, Bernhardt C, Patel P, Carrion M, Rahaman SO, Biswas D. Limiting the pathogenesis of Salmonella Typhimurium with berry phenolic extracts and linoleic acid overproducing Lactobacillus casei. J Microbiol. 2020;58:489-98.

\section{Figures}




\section{a}

th

Newly hatched chicks
Groups:

NT: non-treated

FT: FFC-pretreated

ST: $S$. Enteritidis-infected

FST: FFC-pretreated and S. Enteritidis-infected

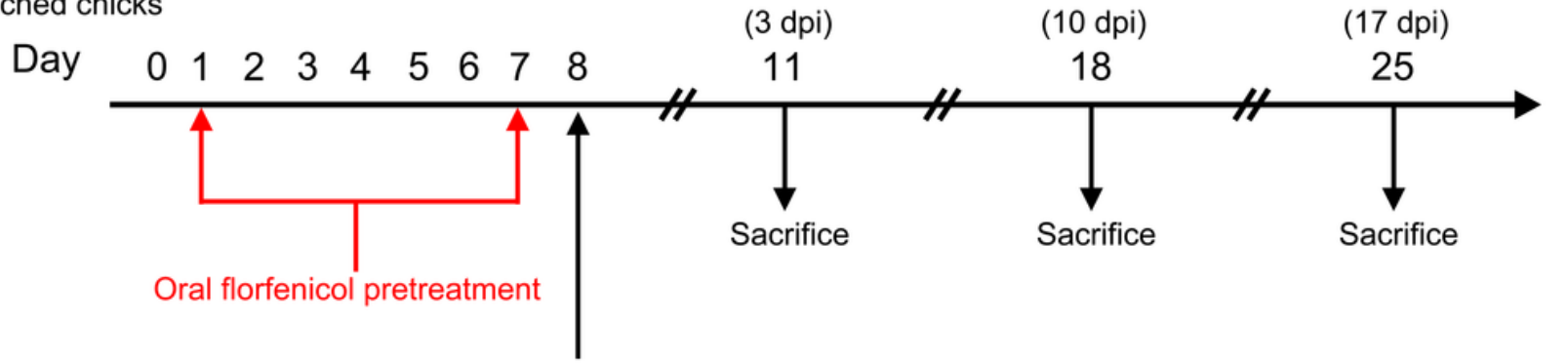

Oral S. Enteritidis infection

b

Cecal content

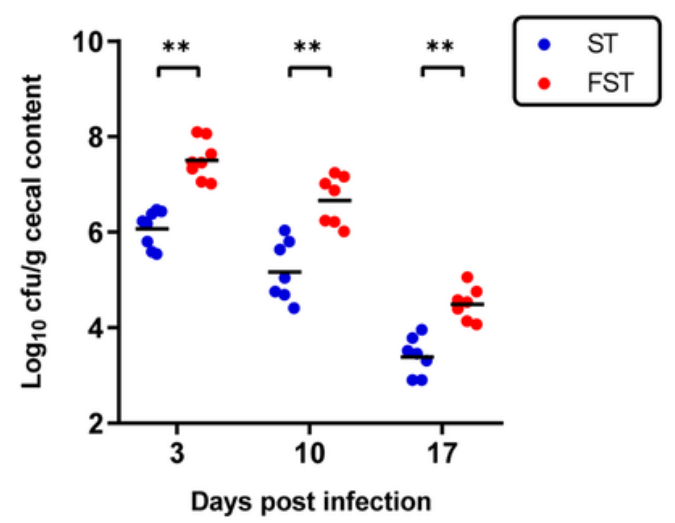

Spleen

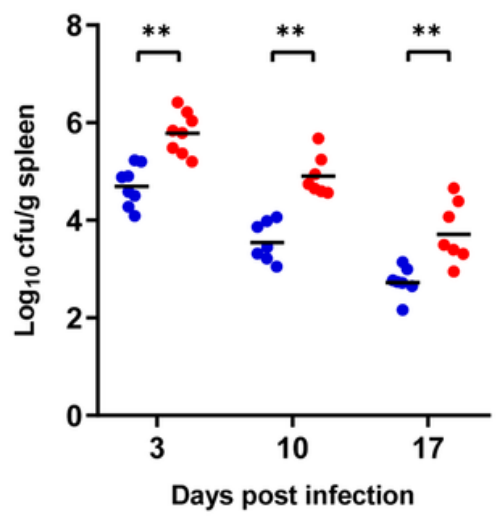

Liver

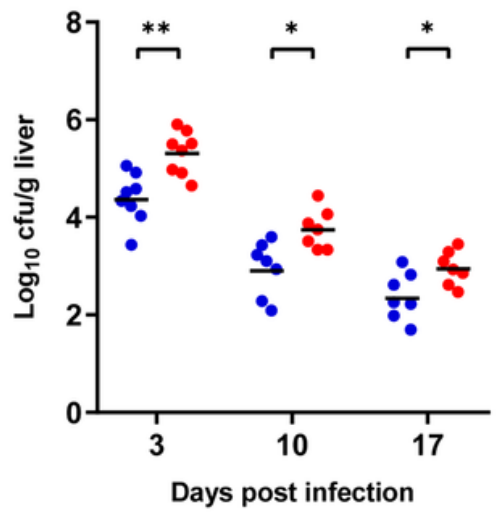

Figure 1

Effect of florfenicol pretreatment on S. Enteritidis infection in neonatal chicks. a Animal experiment design. Newly hatched chicks ( $n=7-8$ chicks in each sampling point) were randomly divided into four groups (NT, FT, ST and FST), which treated with a 7-day treatment $(30 \mathrm{mg} / \mathrm{kg} \mathrm{b.} \mathrm{w.)} \mathrm{of} \mathrm{florfenicol} \mathrm{or}$ infected with $108 \mathrm{cfu}$ of the challenge strain S. Enteritidis by oral gavage. Sampling points for cecal microbiota analysis during infection are indicated. Animals were euthanized, and the S. Enteritidis loads in the cecal contents and organs were determined by plate counting method. b At 3, 10, or 17 days post infection (dpi), chicks were sacrificed and S. Enteritidis loads in the cecal content, spleen, and liver were determined. Bar indicates median. $n s$, not significant $(p \geq 0.05)$; ${ }^{\star} p<0.05 ; * \star p<0.01$; ${ }^{* \star *} p<0.001$; MannWhitney U test. 


\section{NT \\ FT \\ ST \\ FST}
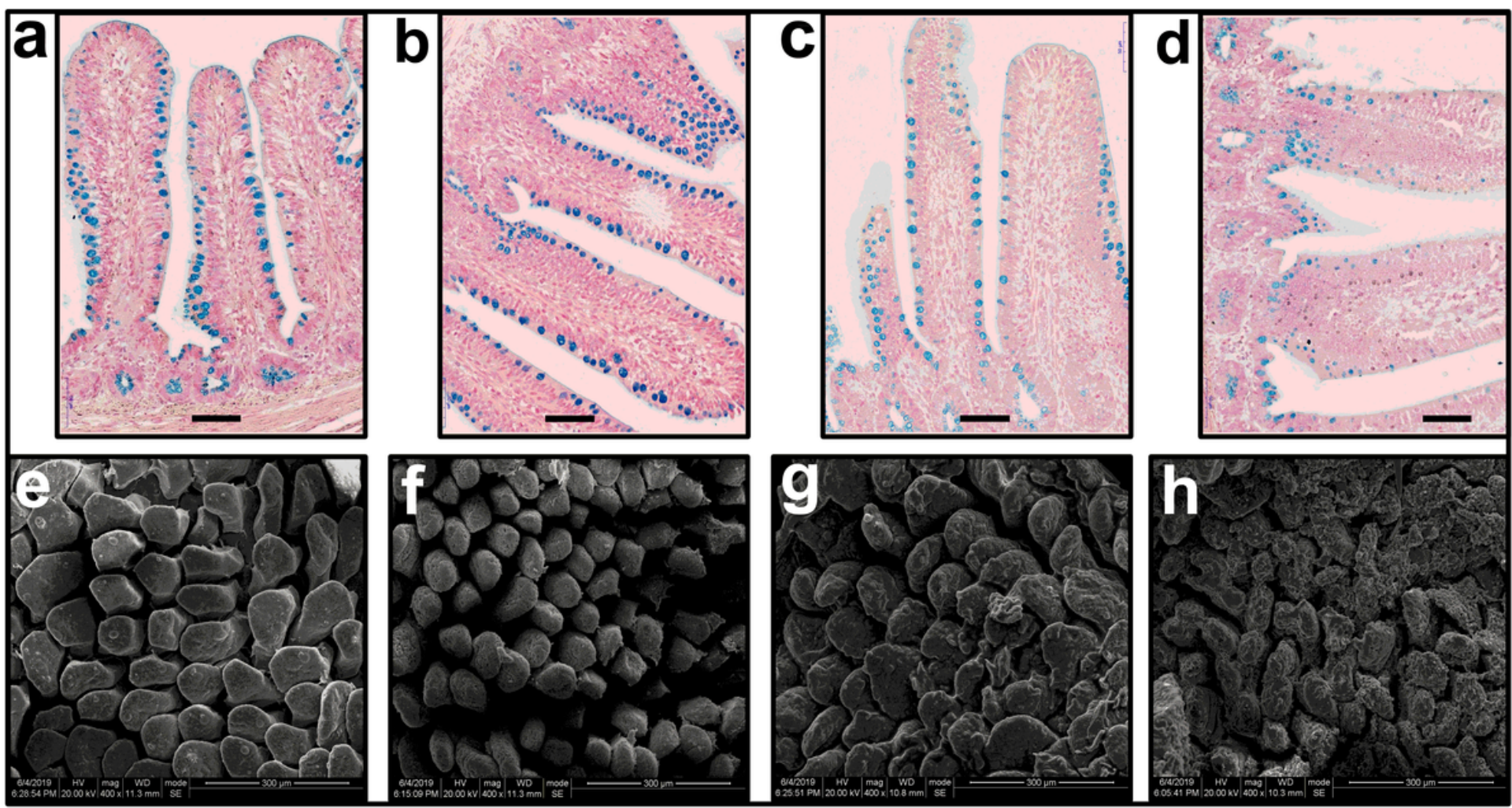

Figure 2

Florfenicol exacerbates the development of Salmonella-induced ileal mucosal injury in neonatal chicks. Chick were randomly divided into four groups: NT (Control group), FT (FFC pretreated group), ST (S. Enteritidis infected group) and FST (FFC pretreated and following S. Enteritidis infection group). All the chicks were infected orally with $~ 108 \mathrm{CFU}$ of S. Enteritidis except the NT and FT groups. After 3 days of infection, the histopathology of the intestinal mucosa was analyzed by Alcian blue staining $(a-d)$ and SEM (e-h). Scale bars, $50 \mu \mathrm{m}(a-d)$; Scale bars, $300 \mu \mathrm{m}(e-h)$. 
a

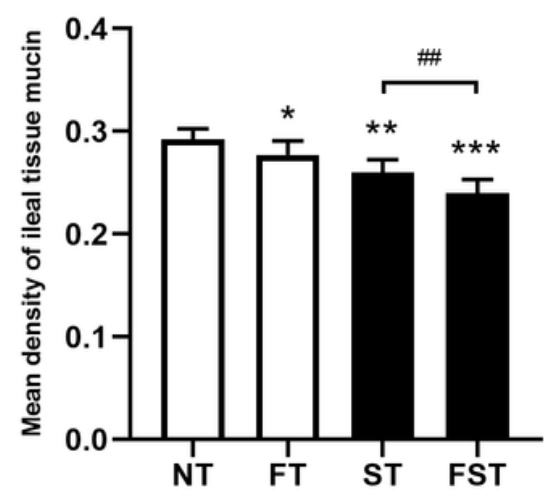

d

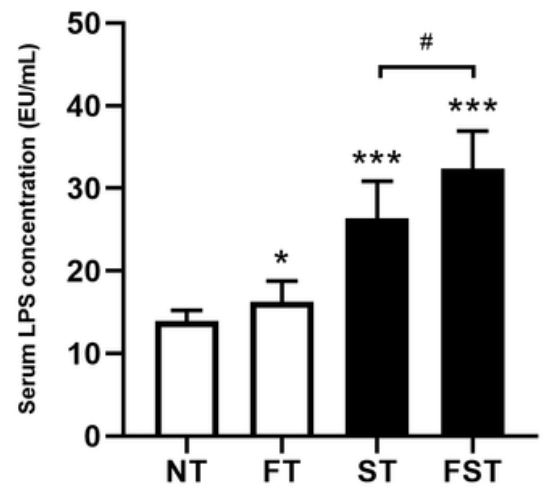

b

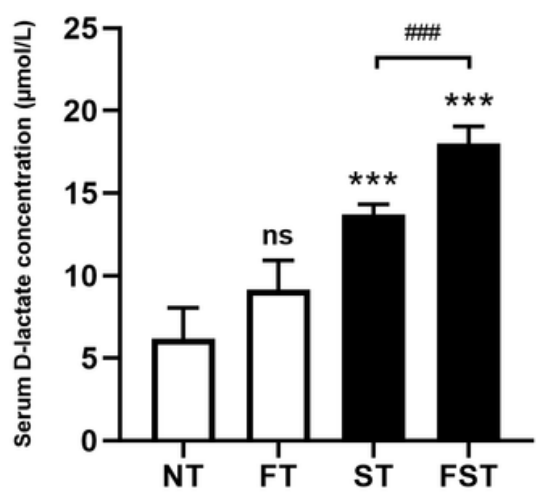

e

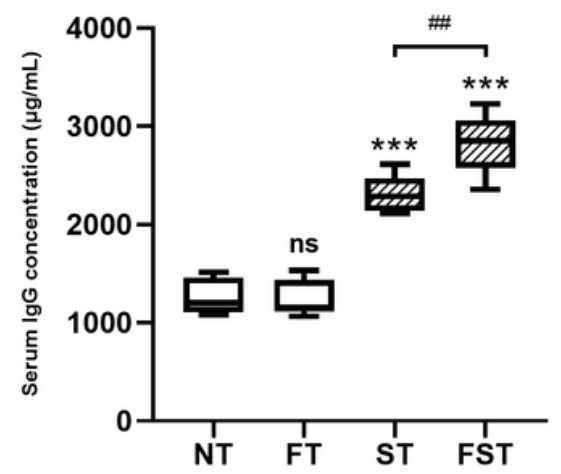

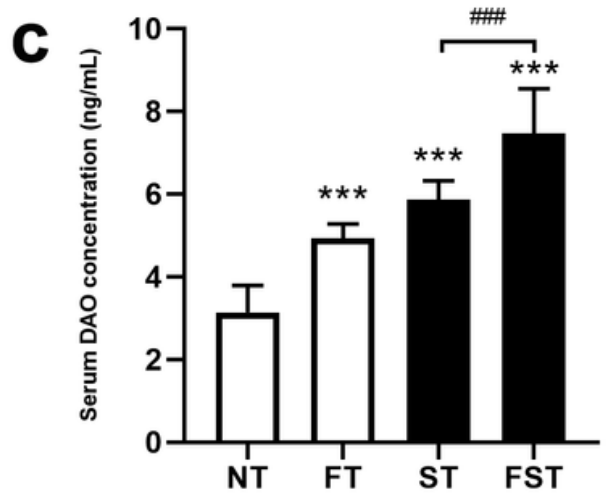

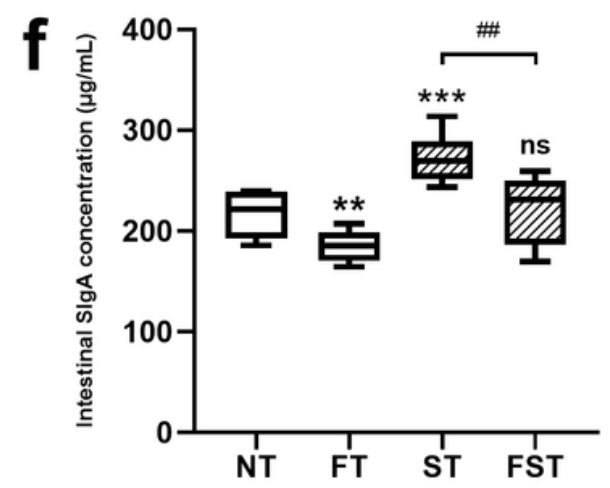

Figure 3

Effect of FFC on Intestinal permeability and immune activity in normal and S. Enteritidis-infected chicks. Mean density of ileal tissue mucin $(n=8 ; a)$, serum D-lactate $(n=8 ; b)$, serum DAO $(n=8 ; c)$, serum LPS $(n=8 ; d)$, serum IgG $(n=8 ; e)$ and intestinal $\operatorname{SIgA}(n=8 ; f)$ were compared among the four groups at 3 dpi. Data are expressed as mean \pm standard deviation were assessed by ANOVA and denoted as follows: ${ }^{\star} \mathrm{P}<0.05,{ }^{*} \mathrm{P}<0.01,{ }^{\star *} \mathrm{P}<0.001, \mathrm{FT}$, ST and FST vs NT; \#P<0.05, \#\#P<0.01, \#\#\#P<0.001, FST vs ST; ns, not significant. NT: Control group. FT: FFC pretreated group. ST: S. Enteritidis infected group. FST: FFC pretreated and following S. Enteritidis infection group. 

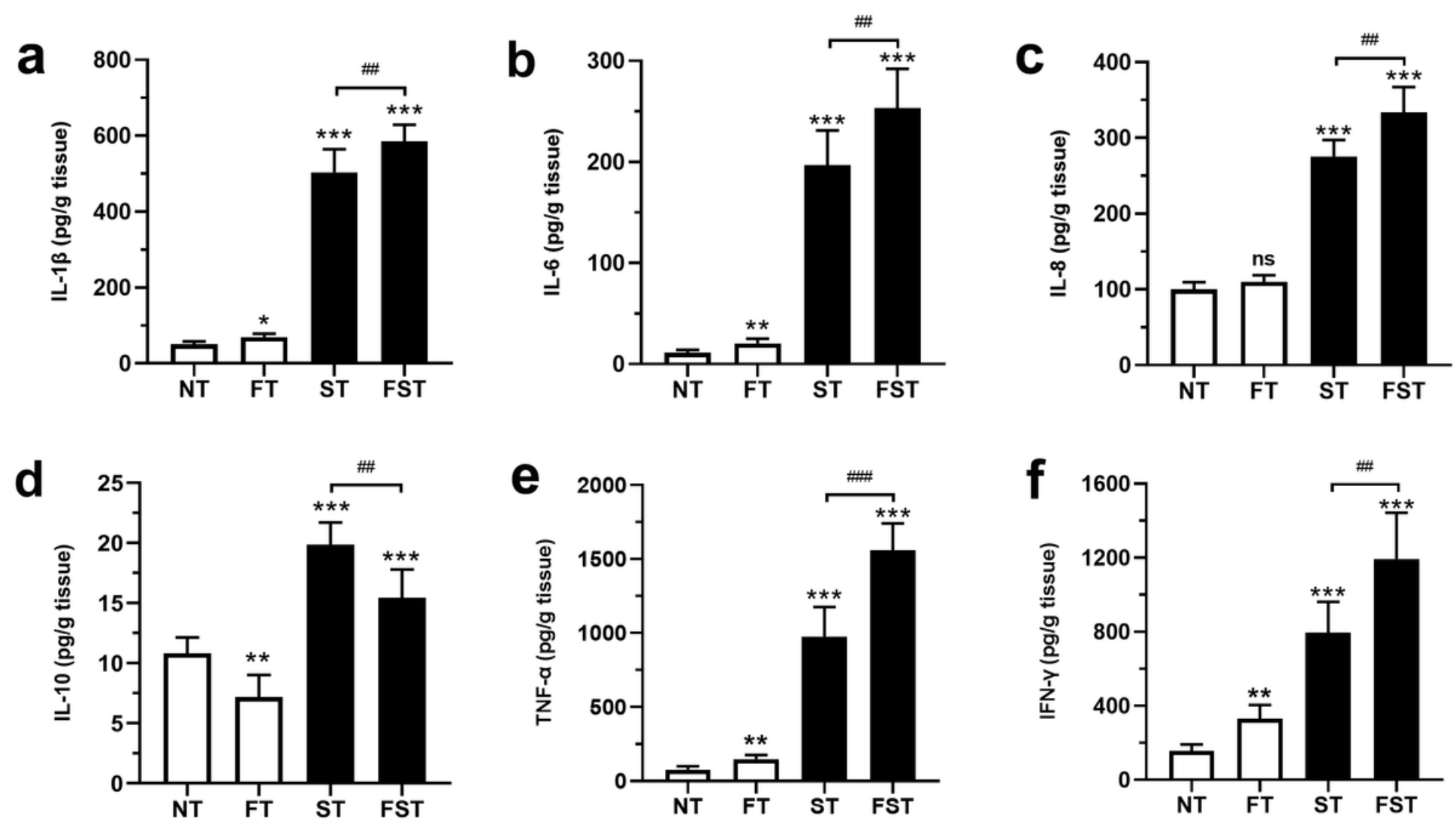

Figure 4

Effect of FFC on the expression of cytokines in ileum of normal and S. Enteritidis-infected chicks. IL-1 $\beta$ ( $\mathrm{n}$ $=8 ; a), I L-6(n=8 ; b), I L-8(n=8 ; c), I L-10(n=8 ; d)$, TNF- $a(n=8 ; e)$ and INF-Y $(n=8 ; f)$ were compared among the four groups at $3 \mathrm{dpi}$. Data are expressed as mean \pm standard deviation were assessed by ANOVA and denoted as follows: ${ }^{*}<<0.05,{ }^{\star} \mathrm{P}<0.01$, ${ }^{* *} \mathrm{P}<0.001, \mathrm{FT}$, ST and FST vs NT; \#P $<0.05$, \#\#P $<0.01$, \#\#\#P<0.001, FST vs ST; ns, not significant. NT: Control group. FT: FFC pretreated group. ST: S. Enteritidis infected group. FST: FFC pretreated and following S. Enteritidis infection group.

\begin{tabular}{|c|c|c|c|c|c|c|c|}
\hline FT & ST & FST & Gene & NT vs FT & NT vs ST & NT vs FS & ST vs FST \\
\hline & & & ZO-1 & ns & * & $\star \star * *$ & * \\
\hline & & & Occludin & ns & * & *** & ** \\
\hline & & & Claudin & * & * & *** & *** \\
\hline & & & MUC2 & ns & ns & * & * \\
\hline & & & TFF2 & ns & ns & ** & * \\
\hline & & & IL-17A & 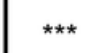 & 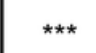 & *** & * \\
\hline & & & IL-22 & ns & * & *** & ns \\
\hline & & & IFN- $\alpha$ & ** & $* \star * *$ & *** & ** \\
\hline
\end{tabular}

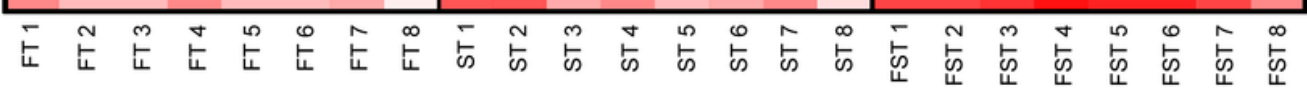

Figure 5 
The gene expression profile in response to FFC pretreatment or S. Enteritidis infection by qRT-PCR at 3dpi. Represented as log2 of the fold change between the treatment group and the control group (NT). Statistical analysis was conducted using one-way ANOVA and Dunnett's multiple comparison test and denoted as follows: ${ }^{*} \mathrm{P}<0.05,{ }^{\star *} \mathrm{P}<0.01,{ }^{*} * \mathrm{P}<0.001$; ns, not significant. NT: Control group. FT: FFC pretreated group. ST: S. Enteritidis infected group. FST: FFC pretreated and following S. Enteritidis infection group.
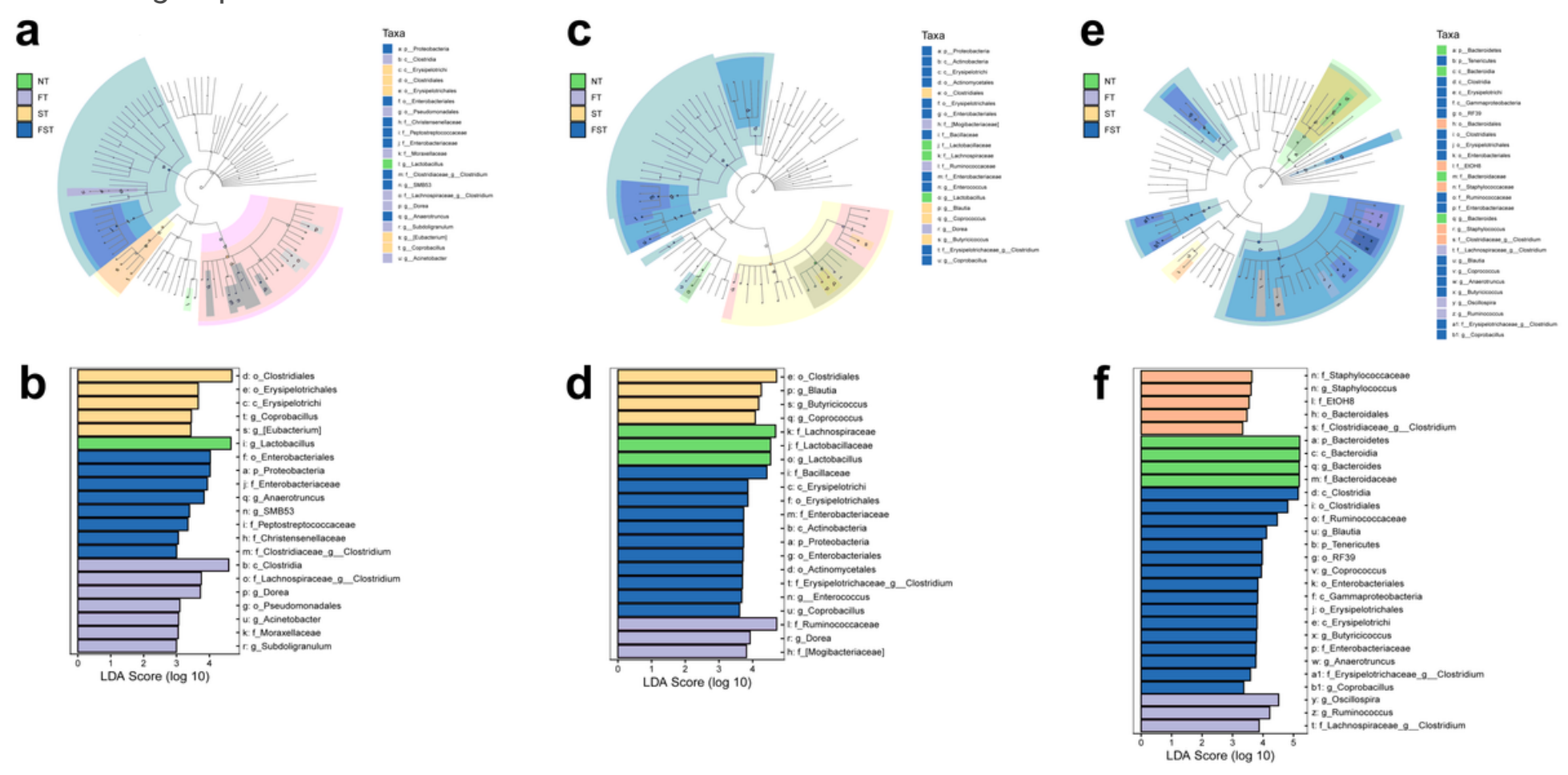

Figure 6

Differences in the gut microbiota of the chicken cecum microbial community were determined using the LefSe analytic method. LefSe plots $(P<0.05$, log LDA score $>3)$ showing microbial strains with significant differences among four groups at 3-(a), 10-(c) and 17-days (e) post infection, respectively. The different groups are represented by different colors, the microbiota that plays an important role in the different groups is represented by nodes of corresponding colors, and the organism markers are indicated by colored circles. From inside to outside, the circles are ordered by species at the level of phylum, class, order, family, and genus. LDA diagram at three sampling points showed in $b, d$ and $f$. Biomarkers with statistical differences are emphasized, with the colors of the histograms representing the respective groups and the lengths representing the LDA score, which is the magnitude of the effects of significantly different species between groups. NT: Control group. FT: FFC pretreated group. ST: S. Enteritidis infected group. FST: FFC pretreated and following S. Enteritidis infection group. 

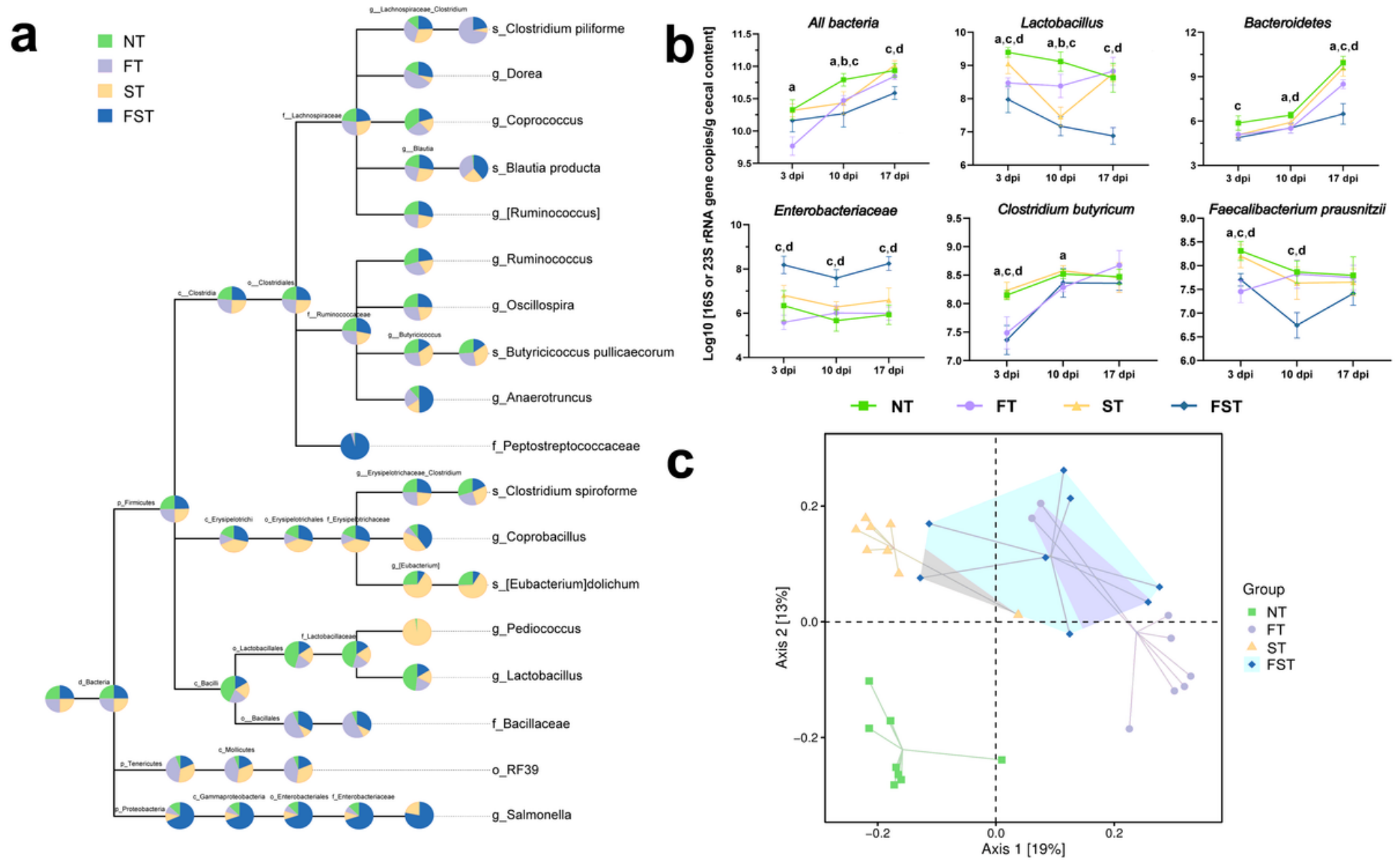

Figure 7

Impact of FFC pretreatment on the cecal microbial communities in response to S. Enteritidis infection at 3 dpi. a The taxonomic cladogram showing the relative abundance of taxa node in each group (relative abundance $>0.1 \%$ ). The larger sector area indicates the higher the abundance of the taxon in the corresponding group. b Quantification of cecal microbiota in different groups at $3 \mathrm{dpi}, 10 \mathrm{dpi}$ and $17 \mathrm{dpi}$ by qPCR of $16 S$ or $23 S$ rRNA gene copy number. $n=7-8$. All bars represent mean $\pm S D$. ${ }^{\star} P<0.05, * * P<$ $0.01, \star \star \star P<0.001 ; n s$, not significant; ANOVA and Dunnett's multiple comparison test. c PCoA for comparison of the changes in bacterial communities of different groups at $3 \mathrm{dpi}$ was generated using the Bray-Curtis distances method. NT: Control group. FT: FFC pretreated group. ST: S. Enteritidis infected group. FST: FFC pretreated and following S. Enteritidis infection group. 


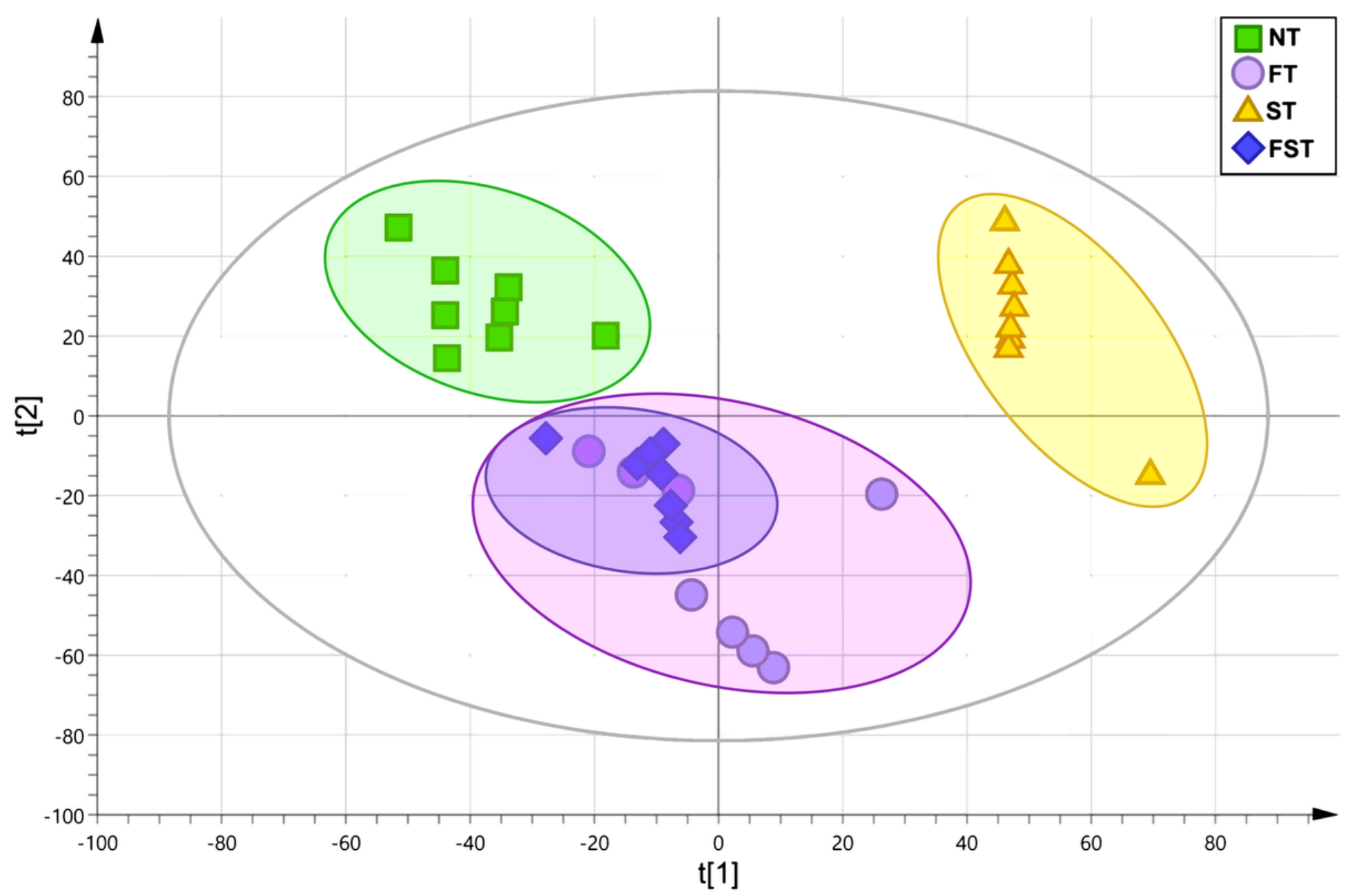

Figure 8

PCA plot of the four groups of the chicks cecal metabolomes. NT: Control group. FT: FFC pretreated group. ST: S. Enteritidis infected group. FST: FFC pretreated and following S. Enteritidis infection group.
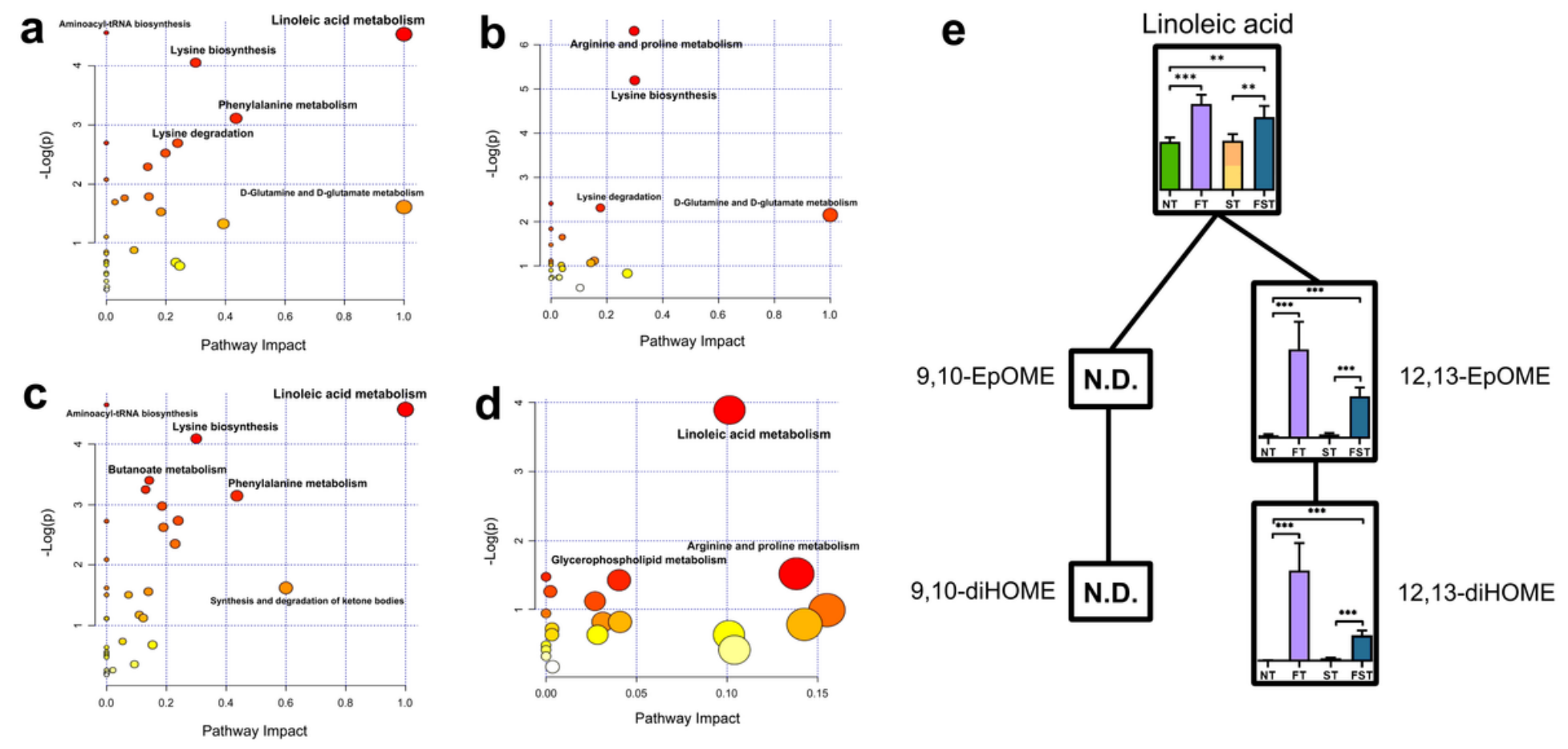


\section{Figure 9}

Statistical comparison of differential metabolites and key metabolic pathways at 3 dpi. Metabolic pathway analysis of biomarker metabolites. Plots showing over-represented metabolic pathways between the FT and NT group (a), ST and NT group (b), FST and NT group (c), FST and ST group (d). The $x$ axis represents the pathway impact, and the $y$ axis represents the pathway enrichment. Larger sizes and darker colors represent higher pathway enrichment levels and higher pathway impact values, respectively. e Linoleic acid metabolomic pathway map. The bar represents the relative amount (mean \pm SD) of a metabolite. N.D., not detected. ${ }^{\star} \mathrm{P}<0.05,{ }^{\star} \mathrm{P}<0.01$, ${ }^{\star \star \star} \mathrm{P}<0.001$; ANOVA and Dunnett's multiple comparison test. NT: Control group. FT: FFC pretreated group. ST: S. Enteritidis infected group. FST: FFC pretreated and following S. Enteritidis infection group.
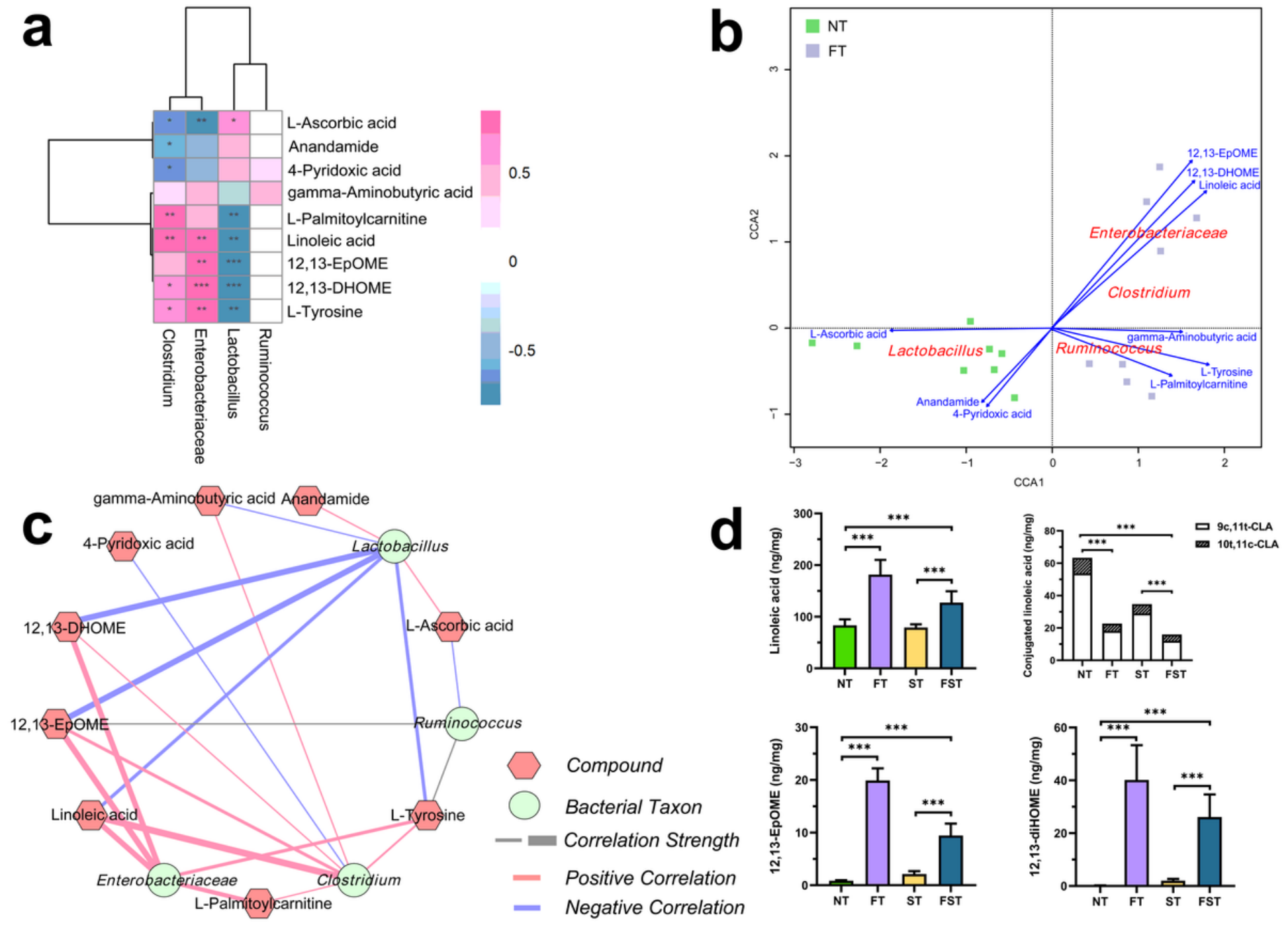

\section{Figure 10}
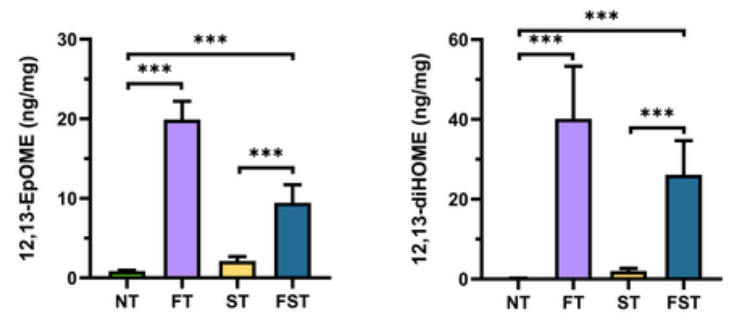

Correlation between key bacterial taxon and differential metabolites, and their absolute abundance. a Spearman correlation between differential metabolites and bacterial taxon was calculated for FT and NT group at 3 dpi $\left({ }^{*} \mathrm{P}<0.05,{ }^{\star} \mathrm{P}<0.01,{ }^{\star *} \mathrm{P}<0.001\right)$. Positive correlation was labelled in red and negative correlation was labelled in blue. b CCA plot of the differential metabolites and bacterial taxon for FT and 
NT group at $3 \mathrm{dpi}$. CCA ordination plot shows the correlations between bacterial community structures and metabolite factors. The correlations between the metabolite factors and key bacterial taxon are represented by the length and angle of the arrows. c Correlation network analysis of the key bacterial taxon and differential metabolites for FT and NT group at $3 \mathrm{dpi}$. The hexagon indicates the metabolite and the circle indicates the bacterial taxon. The lines connecting each node represent the Spearman correlation coefficient values that the red lines represent positive correlation, blue lines represent negative correlation, and the thickness of the edge represents the strength of the correlation. $d$ Concentrations of the key metabolites in cecal content of different group chicks were measured at $3 \mathrm{dpi} . \mathrm{n}=8$. Bars indicate mean $\pm S D$. ${ }^{\star} P<0.05, \star \star P<<0.01$, ${ }^{\star \star \star} P<0.001$; ANOVA and Dunnett's multiple comparison test. NT: Control group. FT: FFC pretreated group. ST: S. Enteritidis infected group. FST: FFC pretreated and following $\mathrm{S}$. Enteritidis infection group. 


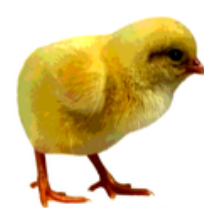

Newly hatched chicks

\section{Groups:}

NT: non-treated

ST: S. Enteritidis-infected

CLA: CLA-pretreated and S. Enteritidis-infected

12,13-diHOME: 12,13-diHOME-pretreated and S. Enteritidis-infected

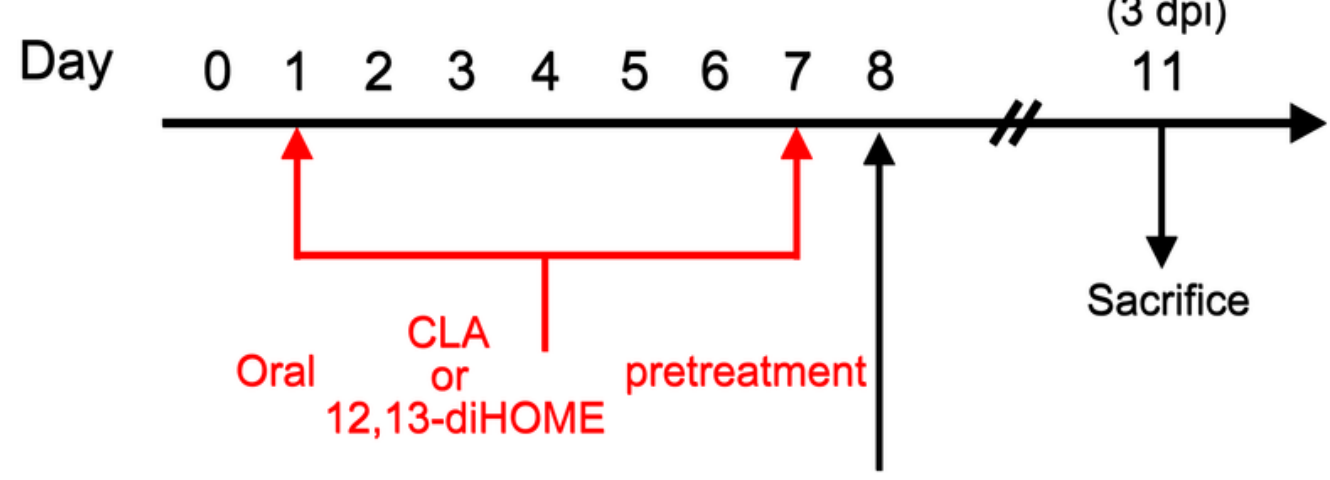

Oral S. Enteritidis infection

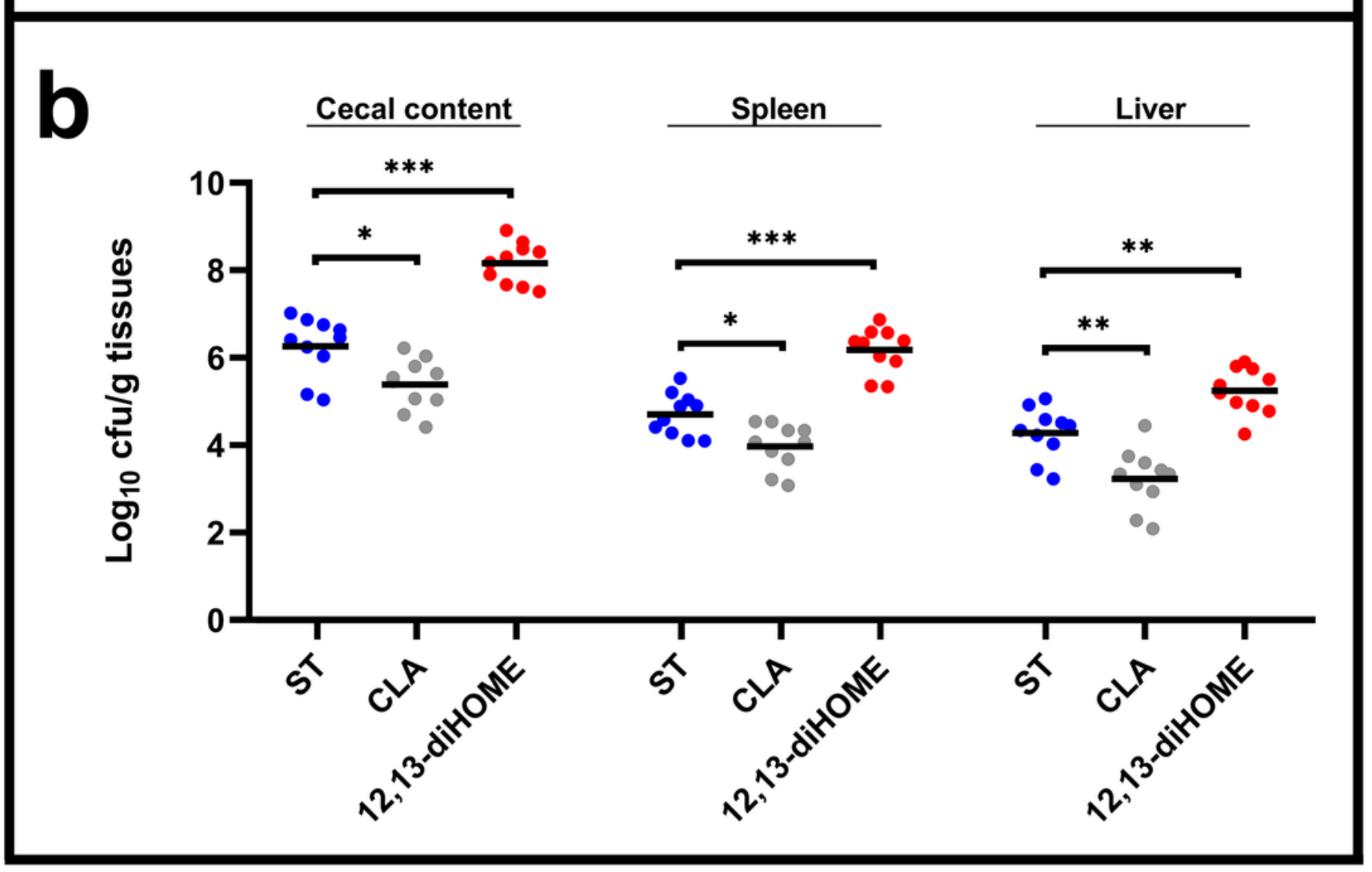

Figure 11

Effect of CLA or 12,13-diHOME pretreatment on S. Enteritidis infection in neonatal chicks. a Animal experiment design. Newly hatched chicks $(n=10)$ were randomly divided into four groups (NT, ST, CLA and 12,13-diHOME), which treated with a 7-day treatment of CLA or 12,13-diHOME and following infected with $\sim 108 \mathrm{cfu}$ of the challenge strain S. Enteritidis by oral gavage. Animals were euthanized, and the S. Enteritidis loads in the cecal contents and organs were determined by plate counting method. b At 3 days 
post infection, chicks were sacrificed and S. Enteritidis loads in the cecal content, spleen, and liver were determined. Bar indicates median. ns, not significant $(p \geq 0.05)$; ${ }^{*} p<0.05 ;{ }^{* *} p<0.01$; ${ }^{* \star *} p<0.001$; MannWhitney U test.
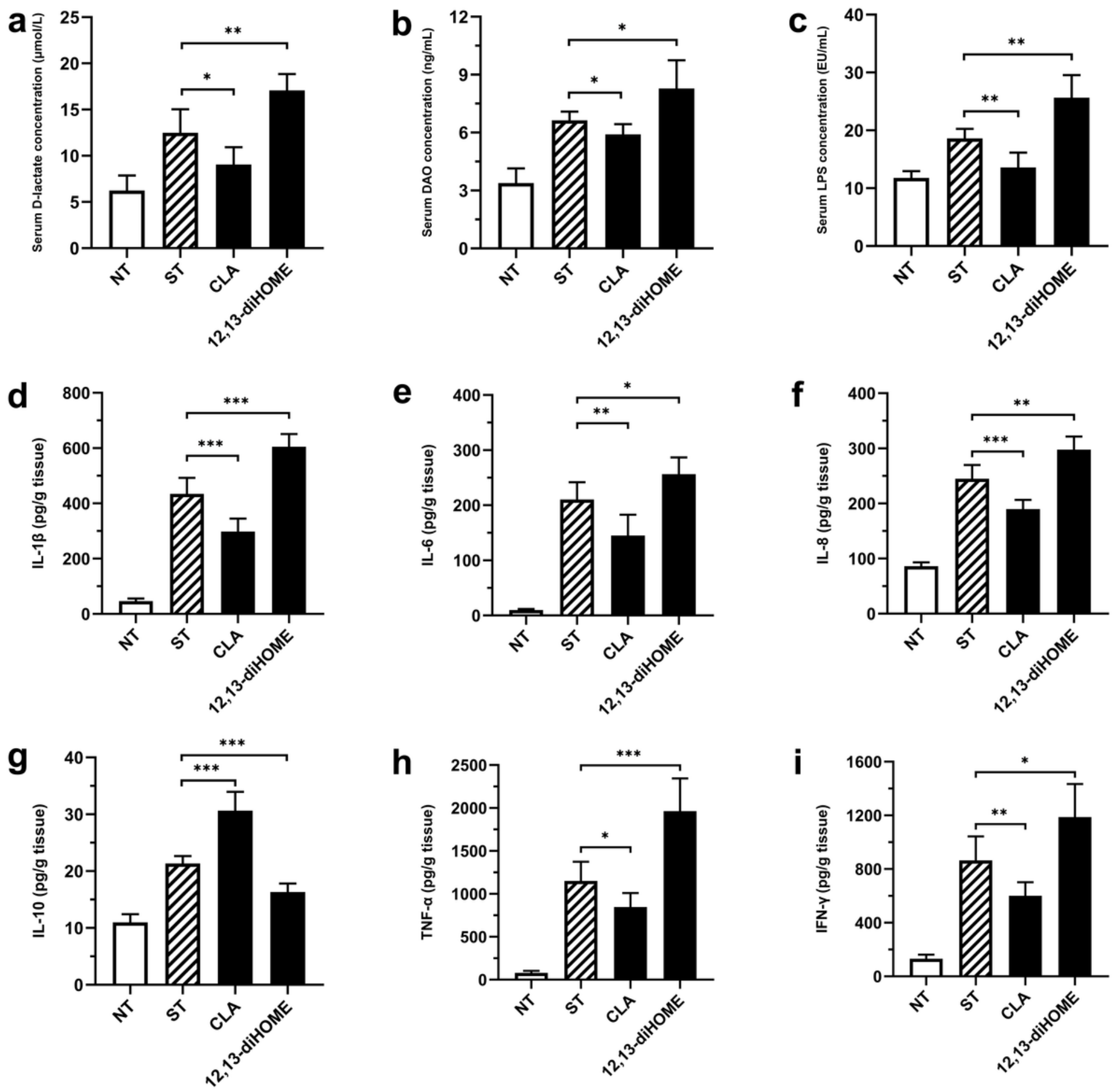

Figure 12

Effect of CLA and 12,13-diHOME pretreatment on Intestinal permeability and expression of cytokines in ileum in S. Enteritidis-infected chicks. Serum D-lactate $(n=10 ; a)$, serum DAO $(n=10 ; b)$, serum LPS $(n=$ $10 ; c), I L-1 \beta(n=10 ; d)$, serum IL-6 ( $n=10 ; e)$, IL-8 $(n=10 ; f), I L-10(n=10 ; g)$, TNF-a $(n=10 ; h)$ and INF-y 
( $n=10$; i) were compared among the four groups at 3 dpi. Data are expressed as mean \pm standard deviation were assessed by ANOVA and denoted as follows: ${ }^{*} P<0.05,{ }^{*} \mathrm{P}<0.01,{ }^{*} * * \mathrm{P}<0.001$. NT: Control group. ST: S. Enteritidis infected group. CLA: CLA pretreated and following S. Enteritidis infection group. 12,13-diHOME: 12,13-diHOME pretreated and following S. Enteritidis infection group.

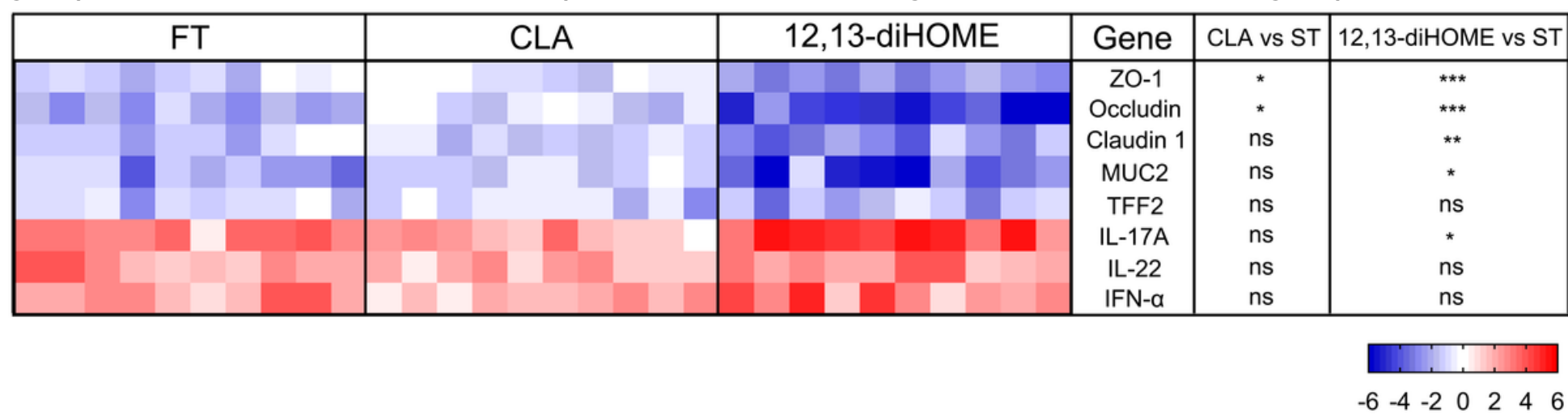

Figure 13

The gene expression profile in response to CLA and 12,13-diHOME pretreatment and following S. Enteritidis infection by qRT-PCR at 3dpi. Represented as log2 of the fold change between the treatment group and the control group (NT). Statistical analysis was conducted using one-way ANOVA and Dunnett's multiple comparison test and denoted as follows: ${ }^{*} P<0.05$, ${ }^{*} P<0.01$, ${ }^{\star *} \mathrm{P} P<0.001$; ns, not significant. NT: Control group. ST: S. Enteritidis infected group. CLA: CLA pretreated and following S. Enteritidis infection group. 12,13-diHOME: 12,13-diHOME pretreated and following S. Enteritidis infection group.

\section{Supplementary Files}

This is a list of supplementary files associated with this preprint. Click to download.

- 2SupplementaryTable3.docx

- 2SupplementaryTable2.docx

- 2SupplementaryTable1.docx

- FigureS11.tif

- FigureS10.tif

- FigureS9.tif

- Figures8.tif

- FigureS7.tif

- Figures6.tif

- FigureS5.tif

- Figures4.tif 
- Figures3.tif

- Figures2.tif

- FigureS1.tif

Page $40 / 40$ 